Article

\title{
Some $(p, q)$-Estimates of Hermite-Hadamard-Type Inequalities for Coordinated Convex and Quasi- Convex Functions
}

\author{
Humaira Kalsoom ${ }^{1,2, *(\mathbb{D})}$, Muhammad Amer ${ }^{3}$, Moin-ud-Din Junjua ${ }^{4} \mathbb{D}$, Sabir Hussain ${ }^{5}$ and \\ Gullnaz Shahzadi 6 \\ 1 School of Mathematical Sciences, Zhejiang University, Hangzhou 310027, China \\ 2 Centre for Advanced Studies in Pure and Applied Mathematics, Bahauddin Zakariya University, \\ Multan 60800, Pakistan \\ 3 Department of Basic Sciences, Deanship of Preparatory Year Program, University of Hail, \\ Hail 2440, Saudi Arabia \\ 4 Department of Mathematics and Statistics, Institute of Southern Punjab, Multan 32100, Pakistan \\ 5 Department of Mathematics, University of Engineering and Technology, Lahore 54890, Pakistan \\ 6 Department of Mechanical Engineering, Ecole de Technologie Superieure, 1100 Notre-Dame W, \\ Montreal, QC H3C 1K3, Canada \\ * Correspondence: humaira87@zju.edu.cn; Tel.: +86-131-8500-1235
}

Received: 19 June 2019; Accepted: 26 July 2019; Published: 31 July 2019

\begin{abstract}
In this paper, we present the preliminaries of $(p, q)$-calculus for functions of two variables. Furthermore, we prove some new Hermite-Hadamard integral-type inequalities for convex functions on coordinates over $[a, b] \times[c, d]$ by using the $(p, q)$-calculus of the functions of two variables. Furthermore, we establish an identity for the right-hand side of the Hermite-Hadamard-type inequalities on coordinates that is proven by using the $(p, q)$-calculus of the functions of two variables. Finally, we use the new identity to prove some trapezoidal-type inequalities with the assumptions of convexity and quasi-convexity on coordinates of the absolute values of the partial derivatives defined in the $(p, q)$-calculus of the functions of two variables.
\end{abstract}

Keywords: Hermite-Hadamard inequality; $(p, q)$-calculus of functions of two variables; partial derivatives in $(p, q)$-calculus of the functions of two variables; multiple integrals in $(p, q)$-calculus of the functions of two variables; Hölder's inequality in $(p, q)$-calculus of the functions of two variables; coordinated convexity; coordinated quasi-convexity inequality

\section{Introduction}

In mathematics, $q$-calculus, also known as quantum calculus, is the study of calculus with no limits. In quantum calculus, we obtain $q$-analogues of mathematical objects that can be recaptured as $q \rightarrow 1^{-}$. The concept was presented by renowned mathematician Euler in the Eighteenth Century, introducing the number in $q$-infinite series defined by Newton. Later on, Jackson [1] introduced the concept of $q$-definite integrals extending the concept of $q$-calculus. Quantum calculus has numerous applications in various fields of mathematics and physics, especially in orthogonal polynomials, number theory, hypergeometric functions, mechanics, and relativity theory. For the background study of quantum calculus theory and the theory of inequalities in quantum calculus, interested, readers are referred to [2-4]. 
The following double inequality is well known in the literature as Hermit-Hadamard's inequality. Let $h: J \subset \mathbb{R} \rightarrow \mathbb{R}$ be convex on $[a, b]$, where $a, b \in J$ with $a<b$ :

$$
h\left(\frac{a+b}{2}\right) \leq \frac{1}{b-a} \int_{a}^{b} h(t) d t \leq \frac{h(a)+h(b)}{2} .
$$

Due to its role in various fields of modern mathematics, such as numerical analysis and functional and mathematical analyses, the validity of Hermit-Hadamard's inequality is important; see [5-7].

The concept of $q$-derivatives over the definite interval $[a, b] \subset \mathbb{R}$ was attended by Tariboon et al. [8,9] and addressed numerous problems on quantum analogues such as the $q$-Hölder inequality, the $q$-Ostrowski inequality, the $q$-Cauchy-Schwarz inequality, the $q$-Grüss-Čebyšev integral inequality, the $q$-Grüss inequality, and other integral inequalities by classical convexity. Most recently, Alp et al. [10] proved the $q$-Hermite-Hadamard inequality and then acquired the generalized $q$-Hermite-Hadamard inequality; also, they studied some integral inequalities, which provide quantum estimates for the left part of the quantum analogue of the $q$-Hermite-Hadamard inequality through $q$-differentiable convex and quasi-convex functions, for more details and interesting applications see References [11-16]. A recent development in the context of the above concept was presented by Tunç and Göv [17-19], named as $(p, q)$-derivatives and $(p, q)$-integrals over $[a, b] \subset \mathbb{R}$. Some well-known results that depend on $(p, q)$-calculus are the $(p, q)$-Minkowski inequality, the $(p, q)$-Hölder inequality, the $(p, q)$-Ostrowski inequality, the $(p, q)$-Cauchy-Schwarz inequality, the $(p, q)$-Grüss-Čebyšev integral inequality, the $(p, q)$-Grüss inequality, and other integral inequalities by classical convexity. Dragomir et al. [20] studied some integral inequalities that provide $(p, q)$ estimates for the right part of the quantum analogue of the Hermit-Hadamard inequality through $(p, q)$ - differentiable convex and quasi-convex functions.

Dragomir [20] proved the following inequalities of the Hermite-Hadamard type for coordinated convex functions on a rectangle from the plane $\mathbb{R}^{2}$.

Theorem 1. Let $h: \triangle=[a, b] \times[c, d] \subset \mathbb{R}^{2} \rightarrow \mathbb{R}$ be convex on the coordinates on $\triangle$ with $a<b$ and $c<d$. Then, one has the inequalities:

$$
\begin{aligned}
& h\left(\frac{a+b}{2}, \frac{c+d}{2}\right) \leq \frac{1}{2}\left[\frac{1}{b-a} \int_{a}^{b} h\left(s, \frac{c+d}{2}\right) d s+\frac{1}{c-d} \int_{c}^{d} h\left(\frac{a+b}{2}, t\right) d t\right] \\
& \leq \frac{1}{(b-a)(d-c)} \int_{a}^{b} \int_{c}^{d} h(s, t) d s d t \leq \frac{1}{4}\left[\frac{1}{b-a}\left(\int_{a}^{b} h(s, c) d s+\int_{a}^{b} h(s, d) d s\right)\right. \\
& \left.+\frac{1}{d-c}\left(\int_{c}^{d} h(a, t) d t+\int_{c}^{d} h(b, t) d t\right)\right] \leq \frac{h(a, c)+h(b, c)+h(a, d)+h(b, d)}{4} .
\end{aligned}
$$

Kunt et al. [21] proved the following $(p, q)$-Hermite-Hadamard inequalities for convex functions by the quantum integral approach:

Theorem 2. Let $0<q<p \leq 1$ and $h:[a, b] \rightarrow \mathbb{R}$ be a convex differentiable function on $[a, b]$. Then, the following integral inequality holds:

$$
h\left(\frac{q a+p b}{p+q}\right) \leq \frac{1}{p(b-a)} \int_{a}^{b p+(1-p) a} h(s)_{a} d_{p, q} s \leq \frac{q h(a)+p h(b)}{p+q} .
$$

Latif et al. [22] for the first time introduced the idea of the $q_{1} q_{2}$-Hermite-Hadamard inequality and obtained the $q_{1} q_{2}$-Hermite-Hadamard-type inequalities for coordinated convex functions on the quantum integral. Furthermore, Alp et al. [23], proved the corrected $q_{1} q_{2}$-Hermite-Hadamard-type inequalities for coordinated convex functions by using quantum calculus. 
Theorem 3. Suppose that $h: \triangle=[a, b] \times[c, d] \subset \mathbb{R}^{2} \rightarrow \mathbb{R}$ is coordinated convex on $\triangle$; the following inequalities hold for all $q_{1}, q_{2} \in(0,1)$ :

$$
\begin{gathered}
h\left(\frac{a q_{1}+b}{1+q_{1}}, \frac{c q_{2}+d}{1+q_{2}}\right) \leq \frac{1}{2}\left[\frac{1}{b-a} \int_{a}^{b} h\left(z, \frac{c q_{2}+d}{1+q_{2}}\right){ }_{a} d_{q_{1}} z+\frac{1}{d-c} \int_{c}^{d} h\left(\frac{a q_{1}+b}{1+q_{1}}, w\right){ }_{c} d_{q_{2}} w\right] \\
\leq \frac{1}{(b-a)(d-c)} \int_{a}^{b} \int_{c}^{d} h(z, w)_{c} d_{q_{2}} w_{a} d_{q_{1}} z \leq \frac{q_{2}}{2\left(1+q_{2}\right)(b-a)} \int_{a}^{b} h(z, c)_{a} d_{q_{1}} z \\
\quad+\frac{1}{2\left(1+q_{2}\right)(b-a)} \int_{a}^{b} h(z, d)_{a} d_{q_{1}} z+\frac{q_{1}}{2\left(1+q_{1}\right)(d-c)} \int_{c}^{d} h(a, w)_{c} d_{q_{2}} w \\
\quad+\frac{1}{2\left(1+q_{1}\right)(d-c)} \int_{c}^{d} h(b, w)_{c} d_{q_{2}} w \leq \frac{q_{1} q_{2} h(a, c)+q_{1} h(a, d)+q_{2} h(b, c)+h(b, d)}{\left(1+q_{1}\right)\left(1+q_{2}\right)} .
\end{gathered}
$$

This paper is organized in the following way. After this Introduction, in Section 2, we define some new concepts regarding the $(p, q)$-calculus of the function of two variables and discuss some properties and examples. Moreover, our aim is to describe the Hermite-Hadamard-type inequalities for functions of two variables using $(p, q)$-calculus, which is a generalization of Theorem 3 presented by Alp et al. [23]. Next, we provide some new estimates for $(p, q)$-analogues of the Hermite-Hadamard-type inequalities of the functions of two variables using convexity and quasi-convexity on coordinates; these results are a generalization of all those results that were presented in [22].

\section{Preliminaries and Main Results}

Definition 1. Let $h: \triangle=[a, b] \times[c, d] \subset \mathbb{R}^{2} \rightarrow \mathbb{R}$ be a continuous function in each variable; let $0<$ $q_{i}<p_{i} \leq 1, i=1,2$; the partial $\left(p_{1}, q_{1}\right)-,\left(p_{2}, q_{2}\right)$ - and $\left(p_{1} p_{2}, q_{1} q_{2}\right)$-derivatives at $(s, t) \in[a, b] \times[c, d]$ are, respectively, defined as:

$$
\begin{aligned}
\frac{{ }_{a} \partial_{p_{1}, q_{1}} h(s, t)}{a} \partial_{p_{1}, q_{1}} s & =\frac{h\left(p_{1} s+\left(1-p_{1}\right) a, t\right)-h\left(q_{1} s+\left(1-q_{1}\right) a, t\right)}{\left(p_{1}-q_{1}\right)(s-a)}, \quad s \neq a \\
\frac{c \partial_{p_{2}, q_{2}} h(s, t)}{c \partial_{p_{2}, q_{2}} t} & =\frac{h\left(s, p_{2} t+\left(1-p_{2}\right) c\right)-h\left(s, q_{2} t+\left(1-q_{2}\right) c\right)}{\left(p_{2}-q_{2}\right)(t-c)}, \quad t \neq c \\
\frac{{ }_{a, c} \partial_{p_{1} p_{2}, q_{1} q_{2}}^{2} h(s, t)}{{ }_{a} \partial_{p_{1}, q_{1}} s_{c} \partial_{p_{2}, q_{2}} t} & =\frac{h\left(q_{1} s+\left(1-q_{1}\right) a, q_{2} t+\left(1-q_{2}\right) c\right)}{\left(p_{1}-q_{1}\right)\left(p_{2}-q_{2}\right)(s-a)(t-b)} \\
& -\frac{h\left(q_{1} s+\left(1-q_{1}\right) a, p_{2} t+\left(1-p_{2}\right) c\right)}{\left(p_{1}-q_{1}\right)\left(p_{2}-q_{2}\right)(s-a)(t-b)} \\
& -\frac{h\left(p_{1} s+\left(1-p_{1}\right) a, q_{2} t+\left(1-q_{2}\right) c\right)}{\left(p_{1}-q_{1}\right)\left(p_{2}-q_{2}\right)(s-a)(t-b)} \\
& +\frac{h\left(p_{1} s+\left(1-p_{1}\right) a, p_{2} t+\left(1-p_{2}\right) c\right)}{\left(p_{1}-q_{1}\right)\left(p_{2}-q_{2}\right)(s-a)(t-b)}, \quad s \neq a, \quad t \neq c .
\end{aligned}
$$

In a similar way, higher order partial derivatives can be defined.

Definition 2 ([24]). For any real number $n$, the $(p, q)$-analogue of $n$ is defined as:

$$
[n]_{p, q}=\frac{p^{n}-q^{n}}{p-q} .
$$


Example 1. Let $h(s, t)=(s-a)^{n}(t-c)^{m}$ and $n, m \in \mathbb{R}_{+}$, then:

$$
\begin{aligned}
& \frac{{ }_{a} \partial_{p_{1}, q_{1}}}{{ }_{a} \partial p_{1, q_{1}} s}\left[(s-a)^{n}(t-c)^{m}\right]=\frac{(t-c)^{m}\left[\left(p_{1}^{n}(s-a)^{n}-q_{1}^{n}(s-a)^{n}\right]\right.}{\left(p_{1}-q_{1}\right)(s-a)} \\
& =\frac{(s-a)^{n}(t-c)^{m}\left(p_{1}^{n}-q_{1}^{n}\right)}{\left(p_{1}-q_{1}\right)(s-a)} \\
& =[n]_{p_{1}, q_{1}}(s-a)^{n-1}(t-c)^{m}, \\
& \frac{{ }_{c} \partial_{p_{2}, q_{2}}}{{ }_{c} \partial_{p_{2}, q_{2}} t}\left[(s-a)^{n}(t-c)^{m}\right]=\frac{(s-a)^{n}\left[\left(p_{2}^{m}(t-c)^{m}-q_{2}^{m}(t-c)^{m}\right]\right.}{\left(p_{2}-q_{2}\right)(t-c)} \\
& =\frac{(s-a)^{n}(t-c)^{m}\left(p_{2}^{m}-q_{2}^{m}\right)}{\left(p_{2}-q_{2}\right)(t-c)} \\
& =[m]_{p_{2}, q_{2}}(s-a)^{n}(t-c)^{m-1}
\end{aligned}
$$

and:

$$
\begin{aligned}
\frac{{ }_{a, c} \partial_{p_{1} p_{2}, q_{1} q_{2}}^{2}}{{ }_{a} \partial_{p_{1}, q_{1}} s_{c} \partial_{p_{2}, q_{2}} t}\left[(s-a)^{n}(t-c)^{m}\right] & =\frac{(s-a)^{n}(t-c)^{m}\left(p_{1}^{n}-q_{1}^{n}\right)\left(p_{2}^{m}-q_{2}^{m}\right)}{\left(p_{1}-q_{1}\right)\left(p_{2}-q_{2}\right)(s-a)(t-a)} \\
& =[n]_{p_{1}, q_{1}}[m]_{p_{2}, q_{2}}(s-a)^{n-1}(t-c)^{m-1},
\end{aligned}
$$

which are the $\left(p_{1} p_{2}, q_{1} q_{2}\right)$-analogues of the usual partial derivatives:

$$
\begin{aligned}
& \frac{\partial}{\partial s}\left[(s-a)^{n}(t-c)^{m}\right]=n(s-a)^{n-1}(t-c)^{m}, \\
& \frac{\partial}{\partial t}\left[(s-a)^{n}(t-c)^{m}\right]=m(s-a)^{n}(t-c)^{m-1}
\end{aligned}
$$

and

$$
\frac{\partial^{2}}{\partial s \partial t}\left[(s-a)^{n}(t-c)^{m}\right]=n m(s-a)^{n-1}(t-c)^{m-1} .
$$

The $(p, q)$-exponential functions are defined as:

$$
\begin{aligned}
& e_{p, q}(x)=\sum_{n=0}^{\infty} p^{\left(\begin{array}{c}
n \\
2
\end{array}\right)} \frac{x^{n}}{[n]_{p, q} !} \\
& E_{p, q}(x)=\sum_{n=0}^{\infty} q^{\left(\frac{n}{2}\right)} \frac{x^{n}}{[n]_{p, q} !} .
\end{aligned}
$$

In the limiting case, as $q \rightarrow 1^{-}$and for $p=1$, the exponential function defined by (3) reduces to the exponential function:

$$
e^{x}=\sum_{n=0}^{\infty} \frac{x^{n}}{n !}
$$

Replacing $x$ by $s-a$ in (3), we obtain:

$$
e_{p, q}(s-a)=\sum_{n=0}^{\infty} p^{\left(\begin{array}{c}
n \\
2
\end{array}\right)} \frac{(s-a)^{n}}{[n]_{p, q} !} .
$$

Example 2. Now, we consider the following exponential function in the $(p, q)$-calculus of the functions of two variables defined by:

$$
e_{p_{1}, q_{1}}(s-a) e_{p_{2}, q_{2}}(t-c)=\sum_{m=0}^{\infty} \sum_{n=0}^{\infty} p_{1}^{\left(\begin{array}{c}
n \\
2
\end{array}\right)} p_{2}^{\left(\begin{array}{c}
m \\
2
\end{array}\right)} \frac{(s-a)^{n}(t-c)^{m}}{[n]_{p_{1}, q_{1} !} ![m]_{p_{2}, q_{2}} !} .
$$


We find the partial derivative of this function with respect to s using the $(p, q)$-calculus of the functions of two variables is:

$$
\begin{aligned}
\frac{{ }_{a} \partial p_{p_{1}, q_{1}}}{a \partial p_{1}, q_{1} s}\left[e_{p_{1}, q_{1}}(s-a) e_{p_{2}, q_{2}}(t-c)\right] & =e_{p_{2}, q_{2}}(t-c) \sum_{n=0}^{\infty} p_{1}^{\left(\begin{array}{c}
n \\
2
\end{array}\right)} \frac{\frac{a \partial p_{1}, q_{1}}{a \partial p_{1}, q_{1} s}\left[(s-a)^{n}\right]}{[n]_{p_{1}, q_{1}} !} \\
& =e_{p_{2}, q_{2}}(t-c) \sum_{n=0}^{\infty} p_{1}^{\left(\begin{array}{c}
n \\
2
\end{array}\right) \frac{[n]_{p_{1}, q_{1}}(s-a)^{n-1}}{[n]_{p_{1}, q_{1}} !}} \\
& =e_{p_{2}, q_{2}}(t-c) \sum_{n=1}^{\infty} p_{1}^{\left(\begin{array}{c}
n \\
2
\end{array}\right)} \frac{(s-a)^{n-1}}{[n-1]_{p_{1}, q_{1} !} !} \\
& =e_{p_{2}, q_{2}}(t-c) \sum_{n=0}^{\infty} p_{1}^{\left(\begin{array}{c}
n+1 \\
2
\end{array}\right)} \frac{(s-a)^{n}}{[n]_{p_{1}, q_{1}} !} \\
& =e_{p_{2}, q_{2}}(t-c) \sum_{n=0}^{\infty} p_{1}^{\left(\begin{array}{c}
n \\
2
\end{array}\right)+\left(\begin{array}{c}
n \\
1
\end{array}\right)} \frac{(s-a)^{n}}{[n]_{p_{1}, q_{1}} !} \\
& =e_{p_{2}, q_{2}}(t-c) \sum_{n=0}^{\infty} p_{1}^{\left(\begin{array}{c}
n \\
2
\end{array}\right)} \frac{(p(s-a))^{n}}{[n]_{p_{1}, q_{1} !} !} \\
& =e_{p_{1}, q_{1}}\left(p_{1}(s-a)\right) e_{p_{2}, q_{2}}(t-c) .
\end{aligned}
$$

Again, taking the partial derivative with respect to ton both sides of Equation (5) using the $(p, q)$-calculus of the functions of two variables, we have:

$$
\begin{aligned}
& \frac{a, c}{{ }_{a} \partial_{p_{1}, q_{1}} s_{c} \partial_{p_{2}, q_{2}} t}\left[e_{p_{1}, q_{1}}(s-a) e_{p_{2}, q_{2}}(t-c)\right] \\
& =e_{p_{1}, q_{1}}\left(p_{1}(s-a)\right) \sum_{m=0}^{\infty} p_{2}^{\left(\begin{array}{c}
m \\
2
\end{array}\right)} \frac{\frac{c \partial_{p_{2}, q_{2}}}{c \partial p_{p_{2}, q_{2}} t}\left[(t-c)^{m}\right]}{[m]_{p_{2}, q_{2}} !} \\
& =e_{p_{1}, q_{1}}\left(p_{1}(s-a)\right) \sum_{m=0}^{\infty} p_{2}^{\left(\begin{array}{c}
m \\
2
\end{array}\right)} \frac{[m]_{p_{2}, q_{2}}(t-c)^{m-1}}{[m]_{p_{1}, q_{2}} !} \\
& =e_{p_{1}, q_{1}}\left(p_{1}(s-a)\right) \sum_{m=1}^{\infty} p_{2}^{\left(\begin{array}{c}
m \\
2
\end{array}\right)} \frac{(t-c)^{m-1}}{[m-1]_{p_{2}, q_{2}} !} \\
& =e_{p_{1}, q_{1}}\left(p_{1}(s-a)\right) \sum_{m=0}^{\infty} p_{2}^{(m+1)} \frac{(t-c)^{m}}{[m]_{p_{2}, q_{2}} !} \\
& =e_{p_{1}, q_{1}}\left(p_{1}(s-a)\right) \sum_{m=0}^{\infty} p_{2}^{\left(\begin{array}{c}
m \\
2
\end{array}\right)+\left(\begin{array}{c}
m \\
1
\end{array}\right)} \frac{(t-c)^{m}}{[m]_{p_{2}, q_{2}} !} \\
& =e_{p_{1}, q_{1}}\left(p_{1}(s-a)\right) \sum_{m=0}^{\infty} p_{2}^{\left(\begin{array}{c}
m \\
2
\end{array}\right)} \frac{\left(p_{2}(t-c)\right)^{m}}{[m]_{p_{2}, q_{2}} !} \\
& =e_{p_{1}, q_{1}}\left(p_{1}(s-a)\right) e_{p_{2}, q_{2}}\left(p_{2}(t-c)\right) \text {, }
\end{aligned}
$$

which is the $\left(p_{1} p_{2}, q_{1} q_{2}\right)$-analogue of the usual partial derivative:

$$
\frac{\partial^{2}}{\partial s \partial t}\left[e^{(s-a)} e^{(t-c)}\right]=e^{(s-a)} e^{(t-c)}
$$

The $(p, q)$-trigonometric functions are defined as:

$$
\begin{aligned}
\sin _{p, q}(x) & =\frac{e_{p, q}(i x)-e_{p, q}(-i x)}{2 i}, \\
\cos _{p, q}(x) & =\frac{e_{p, q}(i x)+e_{p, q}(-i x)}{2} .
\end{aligned}
$$


Replacing $x$ by $s-a$ in (6), we obtain:

$$
\begin{aligned}
& \sin _{p, q}(s-a)=\frac{e_{p_{1}, q_{1}}(i(s-a))-e_{p_{1}, q_{1}}(-i(s-a))}{2 i}, \\
& \cos _{p, q}(s-a)=\frac{e_{p, q}(i(s-a))+e_{p, q}(-i(s-a))}{2} .
\end{aligned}
$$

Example 3. Now, we consider the following trigonometric function of two variables in the $(p, q)$-calculus of the functions of two variables defined by:

$$
\begin{aligned}
& \sin _{p_{1}, q_{1}}(s-a) \cos _{p_{2}, q_{2}}(t-c) \\
& =\left(\frac{e_{p_{1}, q_{1}}(i(s-a))-e_{p_{1}, q_{1}}(-i(s-a))}{2 i}\right)\left(\frac{e_{p_{2}, q_{2}}(i(t-c))+e_{p_{2}, q_{2}}(-i(t-c))}{2}\right) .
\end{aligned}
$$

The partial derivative with respect to s using the $(p, q)$-calculus of functions of two variables is:

$$
\begin{aligned}
& \frac{a \partial_{p_{1}, q_{1}}}{a \partial p_{1}, q_{1} s}\left[\sin _{p_{1}, q_{1}}(s-a) \cos p_{p_{2}, q_{2}}(t-c)\right] \\
& =\left(\frac{\frac{a \partial p_{1}, q_{1}}{a \partial p_{1}, q_{1}} e_{p_{1}, q_{1}}(i(s-a))-\frac{a \partial p_{1}, q_{1}}{a \partial p_{1}, q_{1} s} e_{p_{1}, q_{1}}(-i(s-a))}{2 i}\right) \\
& \times\left(\frac{e_{p_{2}, q_{2}}(i(t-c))+e_{p_{2}, q_{2}}(-i(t-c))}{2}\right) \\
& =\left(\frac{e_{p_{1}, q_{1}}\left(i p_{1}(s-a)\right)+e_{p_{1}, q_{1}}\left(-i p_{1}(s-a)\right)}{2}\right) \\
& \times\left(\frac{e_{p_{2}, q_{2}}(i(t-c))+e_{p_{2}, q_{2}}(-i(t-c))}{2}\right) \\
& =\cos \left(p_{1}(s-a)\right)\left(\frac{e_{p_{2}, q_{2}}(i(t-c))+e_{p_{2}, q_{2}}(-i(t-c))}{2}\right) .
\end{aligned}
$$

Again, taking the partial derivative with respect to t on both sides of Equation (7) using the $(p, q)$-calculus of functions of two variables, we have:

$$
\begin{aligned}
& \frac{a_{,} c \partial_{p_{1} p_{2}, q_{1} q_{2}}^{2}}{{ }_{a} \partial_{p_{1}, q_{1}} s_{c} \partial_{p_{2}, q_{2}} t}\left[\cos _{p_{1}, q_{1}}\left(p_{1}(s-a)\right) \cos _{p_{2}, q_{2}}(t-c)\right] \\
& =\cos \left(p_{1}(s-a)\right)\left(\frac{\frac{c \partial_{p_{2}, q_{2}}}{c \partial_{p_{2}, q_{2} t}} e_{p_{2}, q_{2}}(i(t-c))+\frac{c \partial_{p_{2}, q_{2}}}{c p_{p_{2}, q_{2}} t} e_{p_{2}, q_{2}}(-i(t-c))}{2}\right) \\
& =-\cos \left(p_{1}(s-a)\right)\left(\frac{e_{p_{2}, q_{2}}\left(i p_{2}(t-c)\right)-e_{p_{2}, q_{2}}\left(-i p_{2}(t-c)\right)}{2 i}\right) \\
& =-\cos \left(p_{1}(s-a)\right) \sin \left(p_{2}(t-c)\right)
\end{aligned}
$$

which is the $\left(p_{1} p_{2}, q_{1} q_{2}\right)$-analogue of the usual partial derivative:

$$
\frac{\partial^{2}}{\partial s \partial t}[\sin (s-a) \cos (t-c)]=-\cos (s-a) \sin (t-c) .
$$


Definition 3. Let $h: \triangle=[a, b] \times[c, d] \subset \mathbb{R}^{2} \rightarrow \mathbb{R}$ be a continuous function in each variable; let $0<q_{i}<$ $p_{i} \leq 1$ where $i=1,2$, then the definite $\left(p_{1} p_{2}, q_{1} q_{2}\right)$-integral on $[a, b] \times[c, d]$ is defined as:

$$
\begin{aligned}
& \int_{c}^{t} \int_{a}^{s} h(z, w)_{a} d_{p_{1}, q_{1}} z_{c} d_{p_{2}, q_{2}} w=\left(p_{1}-q_{1}\right)\left(p_{2}-q_{2}\right)(s-a)(t-c) \\
& \times \sum_{m=0}^{\infty} \sum_{n=0}^{\infty} \frac{q_{1}^{n}}{p_{1}^{n+1}} \frac{q_{2}^{m}}{p_{2}^{m+1}} h\left(\frac{q_{1}^{n}}{p_{1}^{n+1}} s+\left(1-\frac{q_{1}^{n}}{p_{1}^{n+1}}\right) a, \frac{q_{2}^{m}}{p_{2}^{m+1}} t+\left(1-\frac{q_{2}^{m}}{p_{2}^{m+1}}\right) c\right)
\end{aligned}
$$

for $(s, t) \in[a, b] \times[c, d]$.

Example 4. Let $h(z, w)=(z-a)^{n}(w-c)^{m}$ for $(s, t) \in[a, b] \times[c, d]$, then we have:

$$
\begin{aligned}
\int_{c}^{t} \int_{a}^{s} h(z, w)_{a} d_{p_{1}, q_{1}} z_{c} d_{p_{2}, q_{2}} w= & \int_{c}^{t} \int_{a}^{s}(z-a)^{n}(w-c)^{m}{ }_{a} d_{p_{1}, q_{1}} z_{c} d_{p_{2}, q_{2}} w \\
= & \left(\left(p_{1}-q_{1}\right)(s-a)^{n+1} p_{1}^{-n-1} \sum_{i=0}^{\infty}\left(\frac{q_{1}^{n+1}}{p_{1}^{n+1}}\right)^{i}\right) \\
& \times\left(\left(p_{2}-q_{2}\right)(t-c)^{m+1} p_{2}^{-m-1} \sum_{j=0}^{\infty}\left(\frac{q_{2}^{m+1}}{p_{2}^{m+1}}\right)^{j}\right) \\
= & \left(\frac{(s-a)^{n+1}}{[n+1]_{p_{1}, q_{1}}}\right)\left(\frac{(t-c)^{m+1}}{[m+1]_{p_{2}, q_{2}}}\right),
\end{aligned}
$$

which is the $\left(p_{1} p_{2}, q_{1} q_{2}\right)$-analogue of the usual integral:

$$
\int_{c}^{t} \int_{a}^{s}(z-a)^{n}(w-c)^{m} d z d w=\left(\frac{(s-a)^{n+1}}{n+1}\right)\left(\frac{(t-c)^{m+1}}{m+1}\right) .
$$

Remark 1. By Definition 3, it directly follows:

1.

$$
\int_{\mathcal{c}}^{t} \int_{a}^{s} h(z, w)_{a} d_{p_{1}, q_{1}} z_{c} d_{p_{2}, q_{2}} w=\int_{a}^{s} \int_{\mathcal{c}}^{t} h(z, w)_{c} d_{p_{2}, q_{2}} w_{a} d_{p_{1}, q_{1}} z
$$

2.

$$
\begin{aligned}
\int_{t_{1}}^{t} \int_{s_{1}}^{s} h(z, w)_{a} d_{p_{1}, q_{1}} z_{c} d_{p_{2}, q_{2}} w= & \int_{c}^{t} \int_{a}^{s} h(z, w)_{a} d_{p_{1}, q_{1}} z_{c} d_{p_{2}, q_{2}} w \\
& -\int_{c}^{t_{1}} \int_{a}^{s} h(z, w)_{a} d_{p_{1}, q_{1}} z_{c} d_{p_{2}, q_{2}} w \\
& -\int_{c}^{t} \int_{a}^{s_{1}} h(z, w)_{a} d_{p_{1}, q_{1}} z_{c} d_{p_{2}, q_{2}} w \\
& +\int_{c}^{t_{1}} \int_{a}^{s_{1}} h(z, w)_{a} d_{p_{1}, q_{1}} z_{c} d_{p_{2}, q_{2}} w
\end{aligned}
$$

for $\left(s_{1}, t_{1}\right) \in(a, s) \times(c, t)$.

3. Setting $q_{i} \rightarrow 1^{-}$and $p_{i}=1$ for $i=1,2$, Definitions 1 and 3 reduce to the classical definitions of partial derivatives and the classical integration of double integrals, respectively.

4. For $p_{i}=1$ where $i=1,2$, Definitions 1 and 3 reduce to Definitions 1 and 2 in [22], respectively. 
Theorem 4. Let $h_{1}, h_{2}: \triangle=[a, b] \times[c, d] \subset \mathbb{R}^{2} \rightarrow \mathbb{R}$ be continuous functions in each variable, then we can prove the following easily:

1.

$$
\frac{a, c}{{ }_{a} \partial_{p_{1}, q_{1}} z_{c} \partial_{p_{2}, q_{2}} w} \int_{c}^{t} \int_{a}^{s} h_{1}(z, w)_{a} d_{p_{1}, q_{1}} z_{c} d_{p_{2}, q_{2}} w=h_{1}(s, t)
$$

2.

$$
\int_{c}^{t} \int_{a}^{s} \frac{{ }_{a, c} \partial_{p_{1} p_{2}, q_{1} q_{2}}^{2} h_{1}(z, w)}{{ }_{c} \partial_{p_{1}, q_{1}} z_{c} \partial_{p_{2}, q_{2}} w}{ }_{a} d_{p_{1}, q_{1}} z_{c} d_{p_{2}, q_{2}} w=h_{1}(s, t) .
$$

For $\left(s_{1}, t_{1}\right) \in(a, s) \times(c, t)$ :

3.

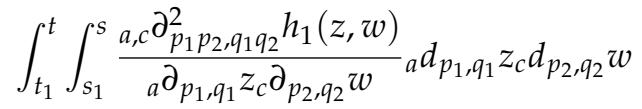

$$
\begin{aligned}
& =h_{1}(s, t)-h_{1}\left(s, t_{1}\right)-h_{1}\left(s_{1}, t\right)+h_{1}\left(s_{1}, t_{1}\right) \text {. }
\end{aligned}
$$

4.

$$
\begin{aligned}
& \int_{c}^{t} \int_{a}^{s}\left[h_{1}(z, w)+h_{2}(z, w)\right]_{a} d_{p_{1}, q_{1}} z_{c} d_{p_{2}, q_{2}} w \\
& =\int_{c}^{t} \int_{a}^{s} h_{1}(z, w)_{a} d_{p_{1}, q_{1}} z_{c} d_{p_{2}, q_{2}} w+\int_{c}^{t} \int_{a}^{s} h_{2}(z, w)_{a} d_{p_{1}, q_{1}} z_{c} d_{p_{2}, q_{2}} w
\end{aligned}
$$

5.

$$
\int_{c}^{t} \int_{a}^{s} \beta h_{1}(z, w)_{a} d_{p_{1}, q_{1}} z_{c} d_{p_{2}, q_{2}} w=\beta \int_{c}^{t} \int_{a}^{s} h_{1}(z, w)_{a} d_{p_{1}, q_{1}} z_{c} d_{p_{2}, q_{2}} w
$$

6.

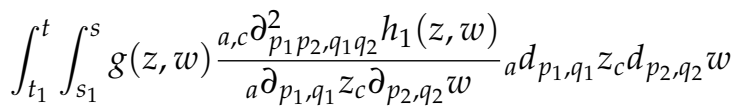

$$
\begin{aligned}
& =g(s, t) h_{1}(s, t)-g\left(s, t_{1}\right) h_{1}\left(s, t_{1}\right)-g\left(s_{1}, t\right) h_{1}\left(s_{1}, t\right)+g\left(s_{1}, t_{1}\right) h_{1}\left(s_{1}, t_{1}\right) \\
& -\int_{t_{1}}^{t} g\left(p_{1} s+\left(1-p_{1}\right) a, q_{2} w+\left(1-q_{2}\right) c\right) \frac{{ }^{c} \partial_{p_{2}, q_{2}} g(s, w)}{c \partial_{p_{2}, q_{2}} w}{ }_{c} d_{p_{2}, q_{2}} w \\
& +\int_{t_{1}}^{t} h_{1}\left(p_{1} s_{1}+\left(1-p_{1}\right) a, q_{2} w+\left(1-q_{2}\right) c\right) \frac{c \partial_{p_{2}, q_{2}} g\left(s_{1}, w\right)}{c{ }_{p_{2}, q_{2}} w}{ } w d_{p_{2}, q_{2}} w \\
& -\int_{s_{1}}^{s} h_{1}\left(q_{1} z+\left(1-q_{1}\right) a, p_{2} t+\left(1-p_{2}\right) c\right) \frac{{ }_{a} \partial \partial_{p_{1}, q_{1}} g(z, t)}{a \partial p_{p_{1}, q_{1}} z}{ }_{a} d_{p_{1}, q_{1}} z \\
& +\int_{s_{1}}^{s} h_{1}\left(q_{1} z+\left(1-q_{1}\right) a, p_{2} t_{1}+\left(1-p_{2}\right) c\right) \frac{a \partial_{p_{1}, q_{1}} g\left(z, t_{1}\right)}{{ }_{a} \partial_{p_{1}, q_{1}} z}{ }_{a} d_{p_{1}, q_{1}} z
\end{aligned}
$$

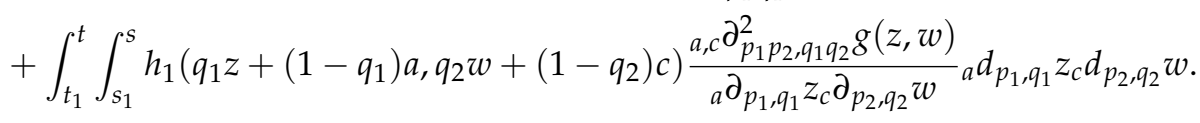

Proof. The proof is directly followed by $\left(p_{1} p_{2}, q_{1} q_{2}\right)$-calculus. We omit the details.

Theorem 5. Let $h:[a, b] \times[c, d] \subset \mathbb{R}^{2} \rightarrow \mathbb{R}$ be convex on the coordinates on $[a, b] \times[c, d]$ for $0<q_{i}<p_{i} \leq 1$ where $i=1,2$, then the following inequalities hold: 


$$
\begin{aligned}
& h\left(\frac{a q_{1}+b p_{1}}{p_{1}+q_{1}}, \frac{c q_{2}+d p_{2}}{p_{2}+q_{2}}\right) \leq \frac{1}{2(b-a) p_{1}} \int_{a}^{p_{1} b+\left(1-p_{1}\right) a} h\left(z, \frac{c q_{2}+d p_{2}}{p_{2}+q_{2}}\right){ }_{a} d_{p_{1}, q_{1}} z \\
& +\frac{1}{2(d-c) p_{2}} \int_{c}^{p_{2} d+\left(1-p_{2}\right) c} h\left(\frac{a q_{1}+b p_{1}}{p_{1}+q_{1}}, w\right){ }_{c} d_{p_{2}, q_{2}} w \\
& \leq \frac{1}{p_{1}(b-a) p_{2}(d-c)} \int_{a}^{p_{1} b+\left(1-p_{1}\right) a} \int_{c}^{p_{2} d+\left(1-p_{2}\right) c} h(z, w)_{a} d_{p_{1}, q_{1}} z_{c} d_{p_{2}, q_{2}} w \\
& \leq \frac{q_{2}}{2 p_{1}\left(p_{2}+q_{2}\right)(b-a)} \int_{a}^{p_{1} b+\left(1-p_{1}\right) a} h(z, c)_{a} d_{p_{1}, q_{1}} z+\frac{p_{2}}{2 p_{1}\left(p_{2}+q_{2}\right)(b-a)} \int_{a}^{p_{1} b+\left(1-p_{1}\right) a} h(z, d)_{a} d_{p_{1}, q_{1}} z \\
& +\frac{q_{1}}{2 p_{2}\left(p_{1}+q_{1}\right)(d-c)} \int_{c}^{p_{2} d+\left(1-p_{2}\right) c} h(a, w)_{c} d_{p_{2}, q_{2}} w+\frac{p_{1}}{2 p_{2}\left(p_{1}+q_{1}\right)(d-c)} \int_{c}^{p_{2} d+\left(1-p_{2}\right) c} h(b, w)_{c} d_{p_{2}, q_{2}} w \\
& \leq \frac{q_{1} q_{2} h(a, c)+p_{2} q_{1} h(a, d)+p_{1} q_{2} h(b, c)+p_{1} p_{2} h(b, d)}{\left(p_{1}+q_{1}\right)\left(p_{2}+q_{2}\right)} .
\end{aligned}
$$

Proof. Since $h:[a, b] \times[c, d] \subset \mathbb{R}^{2} \rightarrow \mathbb{R}$ is a convex function on the coordinates, it follows that the partial mapping $h_{z}:[c, d] \rightarrow \mathbb{R}$ defined by: $h_{z}(w)=h(z, w)$ is convex on $[c, d]$ for $z \in[a, b]$, and hence, by Theorem 2:

$$
h_{z}\left(\frac{c q_{2}+d p_{2}}{p_{2}+q_{2}}\right) \leq \frac{1}{p_{2}(d-c)} \int_{c}^{p_{2} d+\left(1-p_{2}\right) c} h_{z}(w)_{c} d_{p_{2}, q_{2}} w \leq \frac{q_{2} h_{z}(c)+p_{2} h_{z}(d)}{p_{2}+q_{2}} .
$$

Equivalently,

$$
h\left(z, \frac{c q_{2}+d p_{2}}{p_{2}+q_{2}}\right) \leq \frac{1}{p_{2}(d-c)} \int_{c}^{p_{2} d+\left(1-p_{2}\right) c} h(z, w)_{c} d_{p_{2}, q_{2}} w \leq \frac{q_{2} h(z, c)+p_{2} h(z, d)}{p_{2}+q_{2}} .
$$

By $q_{1}$-integrating the inequality (9) on $\left[a, p_{1} b+\left(1-p_{1}\right) a\right]$, we have:

$$
\begin{aligned}
& \frac{1}{p_{1}(b-a)} \int_{a}^{p_{1} b+\left(1-p_{1}\right) a} h\left(z, \frac{c q_{2}+d p_{2}}{p_{2}+q_{2}}\right){ }_{a} d_{p_{1}, q_{1}} z \\
& \leq \frac{1}{p_{1}(b-a) p_{2}(d-c)} \int_{a}^{p_{1} b+\left(1-p_{1}\right) a} \int_{c}^{p_{2} d+\left(1-p_{2}\right) c} h(z, w)_{c} d_{p_{2}, q_{2}} w_{a} d_{p_{1}, q_{1}} z \\
& \leq \frac{1}{p_{1}(b-a)} \int_{a}^{p_{1} b+\left(1-p_{1}\right) a} \frac{q_{2} h(z, c)+p_{2} h(z, d)}{p_{2}+q_{2}}{ }_{a} d_{p_{1}, q_{1}} z .
\end{aligned}
$$

Similarly, consider $h_{w}:[a, b] \rightarrow \mathbb{R}$ defined by $h_{w}(z)=h(z, w)$. Then, obviously, $h_{w}$ is a convex function. Again, the application of Theorem 2 yields the following inequality:

$$
h_{w}\left(\frac{a q_{1}+b p_{1}}{p_{1}+q_{1}}\right) \leq \frac{1}{p_{1}(b-a)} \int_{a}^{p_{1} b+\left(1-p_{1}\right) a} h_{w}(z)_{a} d_{p_{1}, q_{1}} z \leq \frac{q_{1} h_{w}(a)+p_{1} h_{w}(b)}{p_{1}+q_{1}} .
$$

Equivalently,

$$
h\left(\frac{a q_{1}+b p_{1}}{p_{1}+q_{1}}, w\right) \leq \frac{1}{p_{1}(b-a)} \int_{a}^{p_{1} b+\left(1-p_{1}\right) a} h(z, w)_{a} d_{p_{1}, q_{1}} z \leq \frac{q_{1}(a, w)+p_{1} h(b, w)}{p_{1}+q_{1}} .
$$


By $q_{2}$-integrating the inequality (11) on $\left[c, p_{2} d+\left(1-p_{2}\right) c\right]$, we have:

$$
\begin{aligned}
& \frac{1}{p_{2}(d-c)} \int_{c}^{p_{2} d+\left(1-p_{2}\right) c} h\left(\frac{a q_{1}+b p_{1}}{p_{1}+q_{1}}, w\right) c d_{p_{2}, q_{2}} w \\
& \leq \frac{1}{p_{1}(b-a) p_{2}(d-c)} \int_{a}^{p_{1} b+\left(1-p_{1}\right) a} \int_{c}^{p_{2} d+\left(1-p_{2}\right) c} h(z, w)_{c} d_{p_{2}, q_{2}} w_{a} d_{p_{1}, q_{1}} z \\
& \leq \frac{1}{p_{2}(d-c)} \int_{c}^{p_{2} d+\left(1-p_{2}\right) c} \frac{q_{1} h(a, w)+p_{1} h(b, w)}{p_{1}+q_{1}} c d_{p_{2}, q_{2}} w .
\end{aligned}
$$

Adding (10) and (12):

$$
\begin{aligned}
& \frac{1}{2 p_{1}(b-a)} \int_{a}^{p_{1} b+\left(1-p_{1}\right) a} h\left(z, \frac{c q_{2}+d p_{2}}{p_{2}+q_{2}}\right) a d_{p_{1}, q_{1}} z+\frac{1}{2 p_{2}(d-c)} \int_{c}^{p_{2} d+\left(1-p_{2}\right) c} h\left(\frac{a q_{1}+b p_{1}}{p_{1}+q_{1}}, w\right){ }_{c} d_{p_{2}, q_{2}} w \\
& \leq \frac{1}{p_{1}(b-a) p_{2}(d-c)} \int_{a}^{p_{1} b+\left(1-p_{1}\right) a} \int_{c}^{p_{2} d+\left(1-p_{2}\right) c} h(z, w)_{c} d_{p_{2}, q_{2}} w_{a} d_{p_{1}, q_{1}} z \\
& \leq \frac{q_{2}}{2 p_{1}(b-a)\left(p_{2}+q_{2}\right)} \int_{a}^{p_{1} b+\left(1-p_{1}\right) a} h(z, c)_{a} d_{p_{1}, q_{1}} z+\frac{p_{2}}{2 p_{1}(b-a)\left(p_{2}+q_{2}\right)} \int_{a}^{p_{1} b+\left(1-p_{1}\right) a} h(z, d)_{a} d_{p_{1}, q_{1}} z \\
& \leq \frac{q_{1}}{2 p_{2}(d-c)\left(p_{1}+q_{1}\right)} \int_{c}^{p_{2} d+\left(1-p_{2}\right) c} h(a, w)_{c} d_{p_{2}, q_{2}} w+\frac{p_{1}}{2 p_{2}(d-c)\left(p_{1}+q_{1}\right)} \int_{c}^{p_{2} d+\left(1-p_{2}\right) c} h(b, w)_{c} d_{p_{2}, q_{2}} w .
\end{aligned}
$$

Setting $z \rightarrow \frac{a q_{1}+b p_{1}}{p_{1}+q_{1}}$ and $w \rightarrow \frac{c q_{2}+d p_{2}}{p_{2}+q_{2}}$ in (9) and (11) respectively:

$$
\begin{aligned}
& h\left(\frac{a q_{1}+b p_{1}}{p_{1}+q_{1}}, \frac{c q_{2}+d p_{2}}{p_{2}+q_{2}}\right) \leq \frac{1}{p_{2}(d-c)} \int_{c}^{p_{2} d+\left(1-p_{2}\right) c} h\left(\frac{a q_{1}+b p_{1}}{p_{1}+q_{1}}, w\right){ }_{c} d_{p_{2}, q_{2}} w \\
& \leq \frac{q_{2} h\left(\frac{a q_{1}+b p_{1}}{p_{1}+q_{1}}, c\right)+p_{2} h\left(\frac{a q_{1}+b p_{1}}{p_{1}+q_{1}}, d\right)}{p_{2}+q_{2}} \\
& h\left(\frac{a q_{1}+b p_{1}}{p_{1}+q_{1}}, \frac{c q_{2}+d p_{2}}{p_{2}+q_{2}}\right) \leq \frac{1}{p_{1}(b-a)} \int_{a}^{p_{1} b+\left(1-p_{1}\right) a} h\left(z, \frac{c q_{2}+d p_{2}}{p_{2}+q_{2}}\right){ }_{a} d_{p_{1}, q_{1}} z \\
& \leq \frac{q_{1} h\left(a, \frac{c q_{2}+d p_{2}}{p_{2}+q_{2}}\right)+p_{1} h\left(b, \frac{c q_{2}+d p_{2}}{p_{2}+q_{2}}\right)}{p_{1}+q_{1}}
\end{aligned}
$$

and hence, by the addition of (14) and (15), we get:

$$
\begin{aligned}
& h\left(\frac{a q_{1}+b p_{1}}{p_{1}+q_{1}}, \frac{c q_{2}+d p_{2}}{p_{2}+q_{2}}\right) \leq \frac{1}{2(b-a) p_{1}} \int_{a}^{p_{1} b+\left(1-p_{1}\right) a} h\left(z, \frac{c q_{2}+d p_{2}}{p_{2}+q_{2}}\right){ }_{a} d_{p_{1}, q_{1}} z \\
& +\frac{1}{2(d-c) p_{2}} \int_{c}^{p_{2} d+\left(1-p_{2}\right) c} h\left(\frac{a q_{1}+b p_{1}}{p_{1}+q_{1}}, w\right){ }_{c} d_{p_{2}, q_{2}} w \\
& \leq \frac{q_{2} h\left(\frac{a q_{1}+b p_{1}}{p_{1}+q_{1}}, c\right)+p_{2} h\left(\frac{a q_{1}+b p_{1}}{p_{1}+q_{1}}, d\right)}{2\left(p_{2}+q_{2}\right)}+\frac{q_{1} h\left(a, \frac{c q_{2}+d p_{2}}{p_{2}+q_{2}}\right)+p_{1} h\left(b, \frac{c q_{2}+d p_{2}}{p_{2}+q_{2}}\right)}{2\left(p_{1}+q_{1}\right)} .
\end{aligned}
$$

By using Theorem 2 on the right-hand side of (13), we have:

$$
\begin{aligned}
& \frac{q_{2}}{2 p_{1}\left(p_{2}+q_{2}\right)(b-a)} \int_{a}^{p_{1} b+\left(1-p_{1}\right) a} h(z, c)_{a} d_{p_{1}, q_{1}} z \leq \frac{q_{2}}{2\left(p_{2}+q_{2}\right)} \frac{q_{1} h(a, c)+p_{1} h(b, c)}{p_{1}+q_{1}} \\
& \frac{p_{2}}{2 p_{1}\left(p_{2}+q_{2}\right)(b-a)} \int_{a}^{p_{1} b+\left(1-p_{1}\right) a} h(z, d)_{a} d_{p_{1}, q_{1}} z \leq \frac{p_{2}}{2\left(p_{2}+q_{2}\right)} \frac{q_{1} h(a, d)+p_{1} h(b, d)}{p_{1}+q_{1}}
\end{aligned}
$$




$$
\begin{gathered}
\frac{q_{1}}{2 p_{2}\left(p_{1}+q_{1}\right)(d-c)} \int_{c}^{p_{2} d+\left(1-p_{2}\right) c} h(a, w)_{c} d_{p_{2}, q_{2}} w \leq \frac{q_{1}}{2\left(p_{1}+q_{1}\right)} \frac{q_{2} h(a, c)+p_{2} h(a, d)}{p_{2}+q_{2}} \\
\frac{p_{1}}{2 p_{2}\left(p_{1}+q_{1}\right)(d-c)} \int_{c}^{p_{2} d+\left(1-p_{2}\right) c} h(b, w)_{c} d_{p_{2}, q_{2}} w \leq \frac{p_{1}}{2\left(p_{1}+q_{1}\right)} \frac{q_{2} h(b, c)+p_{2} h(b, d)}{p_{2}+q_{2}} .
\end{gathered}
$$

The addition of inequalities (16)-(19) yields the following:

$$
\begin{aligned}
& \frac{q_{2}}{2 p_{1}\left(p_{2}+q_{2}\right)(b-a)} \int_{a}^{p_{1} b+\left(1-p_{1}\right) a} h(z, c)_{a} d_{p_{1}, q_{1}} z+\frac{p_{2}}{2 p_{1}\left(p_{2}+q_{2}\right)(b-a)} \int_{a}^{p_{1} b+\left(1-p_{1}\right) a} h(z, d)_{a} d_{p_{1}, q_{1}} z \\
& +\frac{q_{1}}{2 p_{2}\left(p_{1}+q_{1}\right)(d-c)} \int_{c}^{p_{2} d+\left(1-p_{2}\right) c} h(a, w)_{c} d_{p_{2}, q_{2}} w+\frac{p_{1}}{2 p_{2}\left(p_{1}+q_{1}\right)(d-c)} \int_{c}^{p_{2} d+\left(1-p_{2}\right) c} h(b, w)_{c} d_{p_{2}, q_{2}} w \\
& \leq \frac{q_{1} q_{2} h(a, c)+p_{2} q_{1} h(a, d)+p_{1} q_{2} h(b, c)+p_{1} p_{2} h(b, d)}{\left(p_{1}+q_{1}\right)\left(p_{2}+q_{2}\right)} .
\end{aligned}
$$

Remark 2. 1. Setting $q_{i} \rightarrow 1^{-}$and $p_{i}=1$ for $i=1,2$ then Theorem 5 reduces to Theorem 1 .

2. Also, if $p_{i}=1$ for $i=1,2$ in Theorem 5 , then Theorem 5 reduces to Theorem 3.

Theorem 6 ([22]). Let $\left(x_{m n}\right)_{m, n \in \mathbb{N}}$ and $\left(y_{m n}\right)_{m, n \in \mathbb{N}}$ be sequences of real (or complex) numbers. If $r_{1}^{-1}+r_{2}^{-1}=$ 1 , with $r_{1}, r_{2}>1$, then the following inequality holds:

$$
\sum_{m=0}^{\infty} \sum_{n=0}^{\infty}\left|x_{m n} y_{m n}\right| \leq\left(\sum_{m=0}^{\infty} \sum_{n=0}^{\infty}\left|x_{m n}\right|^{r_{1}}\right)^{\frac{1}{r_{1}}}\left(\sum_{m=0}^{\infty} \sum_{n=0}^{\infty}\left|y_{m n}\right|^{r_{2}}\right)^{\frac{1}{r_{2}}}
$$

provided that all the sums are finite.

Theorem 7. Let $g$ and $h$ be functions defined on $[a, b] \times[c, d]$ and $0<q_{i}<p_{i} \leq 1$ for $i=1$, If $r_{1}^{-1}+r_{2}^{-1}=$ 1 , with $r_{1}, r_{2}>1$, then the following inequality holds:

$$
\begin{aligned}
& \int_{a}^{b} \int_{c}^{d}|g(z, w) h(z, w)|{ }_{c} d_{p_{2}, q_{2}} w_{a} d_{p_{1}, q_{1}} z \leq\left(\int_{a}^{b} \int_{c}^{d}|g(z, w)|^{r_{1}}{ }_{a} d_{p_{1}, q_{1}} z_{c} d_{p_{2}, q_{2}} w\right)^{\frac{1}{r_{1}}} \\
& \times\left(\int_{a}^{b} \int_{c}^{d}|h(z, w)|^{r_{2}}{ }_{a} d_{p_{1}, q_{1}} z_{c} d_{p_{2}, q_{2}} w\right)^{\frac{1}{r_{2}}} .
\end{aligned}
$$

Proof. The proof is directly followed by $\left(p_{1} p_{2}, q_{1} q_{2}\right)$-calculus. We omit the details. 
Lemma 1. Let $h: \triangle \subset \mathbb{R}^{2} \rightarrow \mathbb{R}$ be a function such that $\left(p_{1} p_{2}, q_{1} q_{2}\right)$-derivatives exist on $\triangle^{\mathrm{o}}$ (the interior of $\triangle$ ) and $0<q_{i}<p_{i} \leq 1$ for $i=1,2$. Moreover, if $\frac{a, c \partial p_{1}^{2} p_{2}, q_{1} q_{2} h(z, w)}{{ }_{a} \partial p_{1}, q_{1} z_{c} \partial p_{2}, q_{2} w}$ is continuous and integrable on $[a, b] \times[c, d] \subset \triangle^{\mathrm{o}}$, then:

$$
\begin{aligned}
& \Gamma_{\left(p_{1} p_{2}, q_{1} q_{2}\right)}(a, b, c, d)(h):=\frac{q_{1} q_{2} h(a, c)+p_{2} q_{1} h(a, d)+p_{1} q_{2} h(b, c)+p_{1} p_{2} h(b, d)}{\left(p_{1}+q_{1}\right)\left(p_{2}+q_{2}\right)} \\
& -\frac{q_{2}}{p_{1}\left(p_{2}+q_{2}\right)(b-a)} \int_{a}^{p_{1} b+\left(1-p_{1}\right) a} h(s, c)_{a} d_{p_{1}, q_{1}} s-\frac{p_{2}}{p_{1}\left(p_{2}+q_{2}\right)(b-a)} \int_{a}^{p_{1} b+\left(1-p_{1}\right) a} h(s, d)_{a} d_{p_{1}, q_{1}} s \\
& -\frac{q_{1}}{p_{2}\left(p_{1}+q_{1}\right)(d-c)} \int_{c}^{p_{2} d+\left(1-p_{2}\right) c} h(a, t)_{c} d_{p_{2}, q_{2}} t-\frac{p_{1}}{p_{2}\left(p_{1}+q_{1}\right)(d-c)} \int_{c}^{p_{2} d+\left(1-p_{2}\right) c} h(b, t)_{c} d_{p_{2}, q_{2}} t \\
& +\frac{1}{p_{1}(b-a) p_{2}(d-c)} \int_{a}^{p_{1} b+\left(1-p_{1}\right) a} \int_{c}^{p_{2} d+\left(1-p_{2}\right) c} h(s, t)_{c} d_{p_{2}, q_{2}} t_{a} d_{p_{1}, q_{1}} s \\
& =\frac{q_{1} q_{2}(b-a)(d-c)}{\left(p_{1}+q_{1}\right)\left(p_{2}+q_{2}\right)} \int_{0}^{1} \int_{0}^{1}\left(1-\left(p_{1}+q_{1}\right) z\right)\left(1-\left(p_{2}+q_{2}\right) w\right) \\
& \times \frac{a, c \partial_{p_{1} p_{2}, q_{1} q_{2}}^{2} h((1-z) a+z b,(1-w) c+w d)}{{ }_{a} \partial_{p_{1}, q_{1}} z_{c} \partial_{p_{1}, q_{2}} w}{ }_{0} d_{p_{1}, q_{1}} z_{0} d_{p_{2}, q_{2}} w .
\end{aligned}
$$

Proof. By the Definition 1 of partial $\left(p_{1} p_{2}, q_{1} q_{2}\right)$-derivatives and the Definition 3 of definite $\left(p_{1} p_{2}, q_{1} q_{2}\right)$-integrals, we have:

$$
\begin{aligned}
& \int_{0}^{1} \int_{0}^{1}\left(1-\left(p_{1}+q_{1}\right) z\right)\left(1-\left(p_{2}+q_{2}\right) w\right) \frac{a_{,} c \partial_{p_{1} p_{2}, q_{1} q_{2}}^{2} h((1-z) a+z b,(1-w) c+w d)}{a \partial_{p_{1}, q_{1}} z_{c} \partial_{p_{2}, q_{2}} w}{ }_{0} d_{p_{1}, q_{1}} z_{0} d_{p_{2}, q_{2}} w \\
& =\frac{1}{\left(p_{1}-q_{1}\right)\left(p_{2}-q_{2}\right)(b-a)(d-c)} \int_{0}^{1} \int_{0}^{1} \frac{\left(1-\left(p_{1}+q_{1}\right) z\right)\left(1-\left(p_{2}+q_{2}\right) w\right)}{w z} \\
& \times\left[h\left(z q_{1} b+\left(1-z q_{1}\right) a, w q_{2} d+\left(1-w q_{2}\right) c\right)-h\left(z q_{1} b+\left(1-z q_{1}\right) a, w p_{2} d+\left(1-w p_{2}\right) c\right)\right. \\
& \left.-h\left(z p_{1} b+\left(1-z p_{1}\right) a, w q_{2} d+\left(1-w q_{2}\right) c\right)+h\left(z p_{1} b+\left(1-z p_{1}\right) a, w p_{2} d+\left(1-w p_{2}\right) c\right)\right] d_{p_{1}, q_{1}} z_{0} d_{p_{1}, q_{2}} w \\
& =\frac{1}{(b-a)(d-c)} \sum_{n=0}^{\infty} \sum_{m=0}^{\infty}\left(1-\left(p_{1}+q_{1}\right) \frac{q_{1}^{n}}{p_{1}^{n+1}}\left(1-\left(p_{2}+q_{2}\right) \frac{q_{2}^{m}}{p_{2}^{m+1}}\right)\right.
\end{aligned}
$$




$$
\begin{aligned}
& \times\left[h\left(\frac{q_{1}^{n+1}}{p_{1}^{n+1}} b+\left(1-\frac{q_{1}^{n+1}}{p_{1}^{n+1}}\right) a, \frac{q_{2}^{m+1}}{p_{2}^{m+1}} d+\left(1-\frac{q_{2}^{m+1}}{p_{2}^{m+1}}\right) c\right)\right. \\
& -h\left(\frac{q_{1}^{n+1}}{p_{1}^{n+1}} b+\left(1-\frac{q_{1}^{n+1}}{p_{1}^{n+1}}\right) a, \frac{q_{2}^{m}}{p_{2}^{m}} d+\left(1-\frac{q_{2}^{m}}{p_{2}^{m}}\right) c\right) \\
& -h\left(\frac{q_{1}^{n}}{p_{1}^{n}} b+\left(1-\frac{q_{1}^{n}}{p_{1}^{n}}\right) a, \frac{q_{2}^{m+1}}{p_{2}^{m+1}} d+\left(1-\frac{q_{2}^{m+1}}{p_{2}^{m+1}}\right) c\right) \\
& \left.+h\left(\frac{q_{1}^{n}}{p_{1}^{n}} b+\left(1-\frac{q_{1}^{n}}{p_{1}^{n}}\right) a, \frac{q_{2}^{m}}{p_{2}^{m}} d+\left(1-\frac{q_{2}^{m}}{p_{2}^{m}}\right) c\right)\right] \\
& =\frac{1}{(b-a)(d-c)} \sum_{n=1}^{\infty} \sum_{m=1}^{\infty} h\left(\frac{q_{1}^{n}}{p_{1}^{n}} b+\left(1-\frac{q_{1}^{n}}{p_{1}^{n}}\right) a, \frac{q_{2}^{m}}{p_{2}^{m}} d+\left(1-\frac{q_{2}^{m}}{p_{2}^{m}}\right) c\right) \\
& -\frac{1}{(b-a)(d-c)} \sum_{n=1}^{\infty} \sum_{m=0}^{\infty} h\left(\frac{q_{1}^{n}}{p_{1}^{n}} b+\left(1-\frac{q_{1}^{n}}{p_{1}^{n}}\right) a, \frac{q_{2}^{m}}{p_{2}^{m}} d+\left(1-\frac{q_{2}^{m}}{p_{2}^{m}}\right) c\right) \\
& -\frac{1}{(b-a)(d-c)} \sum_{n=0}^{\infty} \sum_{m=1}^{\infty} h\left(\frac{q_{1}^{n}}{p_{1}^{n}} b+\left(1-\frac{q_{1}^{n}}{p_{1}^{n}}\right) a, \frac{q_{2}^{m}}{p_{2}^{m}} d+\left(1-\frac{q_{2}^{m}}{p_{2}^{m}}\right) c\right) \\
& +\frac{1}{(d-c)(b-a)} \sum_{n=0}^{\infty} \sum_{m=0}^{\infty} h\left(\frac{q_{1}^{n}}{p_{1}^{n}} b+\left(1-\frac{q_{1}^{n}}{p_{1}^{n}}\right) a, \frac{q_{2}^{m}}{p_{2}^{m}} d+\left(1-\frac{q_{2}^{m}}{p_{2}^{m}}\right) c\right) \\
& -\frac{\left(p_{1}+q_{1}\right)}{q_{1}(b-a)(d-c)} \sum_{n=1}^{\infty} \sum_{m=1}^{\infty} \frac{q_{1}^{n}}{p_{1}^{n}} h\left(\frac{q_{1}^{n}}{p_{1}^{n}} b+\left(1-\frac{q_{1}^{n}}{p_{1}^{n}}\right) a, \frac{q_{2}^{m}}{p_{2}^{m}} d+\left(1-\frac{q_{2}^{m}}{p_{2}^{m}}\right) c\right) \\
& +\frac{\left(p_{1}+q_{1}\right)}{q_{1}(b-a)(d-c)} \sum_{n=1}^{\infty} \sum_{m=0}^{\infty} \frac{q_{1}^{n}}{p_{1}^{n}} h\left(\frac{q_{1}^{n}}{p_{1}^{n}} b+\left(1-\frac{q_{1}^{n}}{p_{1}^{n}}\right) a, \frac{q_{2}^{m}}{p_{2}^{m}} d+\left(1-\frac{q_{2}^{m}}{p_{2}^{m}}\right) c\right) \\
& +\frac{\left(p_{1}+q_{1}\right)}{p_{1}(b-a)(d-c)} \sum_{n=0}^{\infty} \sum_{m=1}^{\infty} \frac{q_{1}^{n}}{p_{1}^{n}} h\left(\frac{q_{1}^{n}}{p_{1}^{n}} b+\left(1-\frac{q_{1}^{n}}{p_{1}^{n}}\right) a, \frac{q_{2}^{m}}{p_{2}^{m}} d+\left(1-\frac{q_{2}^{m}}{p_{2}^{m}}\right) c\right) \\
& -\frac{\left(p_{1}+q_{1}\right)}{p_{1}(b-a)(d-c)} \sum_{n=0}^{\infty} \sum_{m=0}^{\infty} \frac{q_{1}^{n}}{p_{1}^{n}} h\left(\frac{q_{1}^{n}}{p_{1}^{n}} b+\left(1-\frac{q_{1}^{n}}{p_{1}^{n}}\right) a, \frac{q_{2}^{m}}{p_{2}^{m}} d+\left(1-\frac{q_{2}^{m}}{p_{2}^{m}}\right) c\right) \\
& -\frac{\left(p_{2}+q_{2}\right)}{q_{2}(b-a)(d-c)} \sum_{n=1}^{\infty} \sum_{m=1}^{\infty} \frac{q_{2}^{m}}{p_{2}^{m}} h\left(\frac{q_{1}^{n}}{p_{1}^{n}} b+\left(1-\frac{q_{1}^{n}}{p_{1}^{n}}\right) a, \frac{q_{2}^{m}}{p_{2}^{m}} d+\left(1-\frac{q_{2}^{m}}{p_{2}^{m}}\right) c\right) \\
& +\frac{\left(p_{2}+q_{2}\right)}{p_{2}(b-a)(d-c)} \sum_{n=1}^{\infty} \sum_{m=0}^{\infty} \frac{q_{2}^{m}}{p_{2}^{m}} h\left(\frac{q_{1}^{n}}{p_{1}^{n}} b+\left(1-\frac{q_{1}^{n}}{p_{1}^{n}}\right) a, \frac{q_{2}^{m}}{p_{2}^{m}} d+\left(1-\frac{q_{2}^{m}}{p_{2}^{m}}\right) c\right) \\
& +\frac{\left(p_{2}+q_{2}\right)}{q_{2}(b-a)(d-c)} \sum_{n=0}^{\infty} \sum_{m=1}^{\infty} \frac{q_{2}^{m}}{p_{2}^{m}} h\left(\frac{q_{1}^{n}}{p_{1}^{n}} b+\left(1-\frac{q_{1}^{n}}{p_{1}^{n}}\right) a, \frac{q_{2}^{m}}{p_{2}^{m}} d+\left(1-\frac{q_{2}^{m}}{p_{2}^{m}}\right) c\right) \\
& -\frac{\left(p_{2}+q_{2}\right)}{p_{2}(b-a)(d-c)} \sum_{n=0}^{\infty} \sum_{m=0}^{\infty} \frac{q_{2}^{m}}{p_{2}^{m}} h\left(\frac{q_{1}^{n}}{p_{1}^{n}} b+\left(1-\frac{q_{1}^{n}}{p_{1}^{n}}\right) a, \frac{q_{2}^{m}}{p_{2}^{m}} d+\left(1-\frac{q_{2}^{m}}{p_{2}^{m}}\right) c\right) \\
& +\frac{\left(p_{1}+q_{1}\right)\left(p_{2}+q_{2}\right)}{q_{1} q_{2}(b-a)(d-c)} \sum_{n=1}^{\infty} \sum_{m=1}^{\infty} \frac{q_{1}^{n}}{p_{1}^{n}} \frac{q_{2}^{m}}{p_{2}^{m}} h\left(\frac{q_{1}^{n}}{p_{1}^{n}} b+\left(1-\frac{q_{1}^{n}}{p_{1}^{n}}\right) a, \frac{q_{2}^{m}}{p_{2}^{m}} d+\left(1-\frac{q_{2}^{m}}{p_{2}^{m}}\right) c\right) \\
& -\frac{\left(p_{1}+q_{1}\right)\left(p_{2}+q_{2}\right)}{p_{2} q_{1}(b-a)(d-c)} \sum_{n=1}^{\infty} \sum_{m=0}^{\infty} \frac{q_{1}^{n}}{p_{1}^{n}} \frac{q_{2}^{m}}{p_{2}^{m}} h\left(\frac{q_{1}^{n}}{p_{1}^{n}} b+\left(1-\frac{q_{1}^{n}}{p_{1}^{n}}\right) a, \frac{q_{2}^{m}}{p_{2}^{m}} d+\left(1-\frac{q_{2}^{m}}{p_{2}^{m}}\right) c\right) \\
& -\frac{\left(p_{1}+q_{1}\right)\left(p_{1}+q_{2}\right)}{p_{1} q_{2}(b-a)(d-c)} \sum_{n=0}^{\infty} \sum_{m=1}^{\infty} \frac{q_{1}^{n}}{p_{1}^{n}} \frac{q_{2}^{m}}{p_{2}^{m}} h\left(\frac{q_{1}^{n}}{p_{1}^{n}} b+\left(1-\frac{q_{1}^{n}}{p_{1}^{n}}\right) a, \frac{q_{2}^{m}}{p_{2}^{m}} d+\left(1-\frac{q_{2}^{m}}{p_{2}^{m}}\right) c\right) \\
& +\frac{\left(p_{1}+q_{1}\right)\left(p_{2}+q_{2}\right)}{p_{1} p_{2}(b-a)(d-c)} \sum_{n=0}^{\infty} \sum_{m=0}^{\infty} \frac{q_{1}^{n}}{p_{1}^{n}} \frac{q_{2}^{m}}{p_{2}^{m}} h\left(\frac{q_{1}^{n}}{p_{1}^{n}} b+\left(1-\frac{q_{1}^{n}}{p_{1}^{n}}\right) a, \frac{q_{2}^{m}}{p_{2}^{m}} d+\left(1-\frac{q_{2}^{m}}{p_{2}^{m}}\right) c\right) \text {. }
\end{aligned}
$$


We observe that:

$$
\begin{aligned}
& \frac{1}{(b-a)(d-c)} \sum_{n=1}^{\infty} \sum_{m=1}^{\infty} h\left(\frac{q_{1}^{n}}{p_{1}^{n}} b+\left(1-\frac{q_{1}^{n}}{p_{1}^{n}}\right) a, \frac{q_{2}^{m}}{p_{2}^{m}} d+\left(1-\frac{q_{2}^{m}}{p_{2}^{m}}\right) c\right) \\
& =\frac{h(a, c)}{(b-a)(d-c)}+\frac{1}{(b-a)(d-c)} \sum_{n=1}^{\infty} \sum_{m=1}^{\infty} h\left(\frac{q_{1}^{n}}{p_{1}^{n}} b+\left(1-\frac{q_{1}^{n}}{p_{1}^{n}}\right) a, \frac{q_{2}^{m}}{p_{2}^{m}} d+\left(1-\frac{q_{2}^{m}}{p_{2}^{m}}\right) c\right) \text {, } \\
& -\frac{1}{(b-a)(d-c)} \sum_{n=1}^{\infty} \sum_{m=0}^{\infty} h\left(\frac{q_{1}^{n}}{p_{1}^{n}} b+\left(1-\frac{q_{1}^{n}}{p_{1}^{n}}\right) a, \frac{q_{2}^{m}}{p_{2}^{m}} d+\left(1-\frac{q_{2}^{m}}{p_{2}^{m}}\right) c\right) \\
& =-\frac{h(a, d)}{(b-a)(d-c)}-\frac{1}{(b-a)(d-c)} \sum_{n=1}^{\infty} \sum_{m=1}^{\infty} h\left(\frac{q_{1}^{n}}{p_{1}^{n}} b+\left(1-\frac{q_{1}^{n}}{p_{1}^{n}}\right) a, \frac{q_{2}^{m}}{p_{2}^{m}} d+\left(1-\frac{q_{2}^{m}}{p_{2}^{m}}\right) c\right) \text {, } \\
& -\frac{1}{(b-a)(d-c)} \sum_{n=0}^{\infty} \sum_{m=1}^{\infty} h\left(\frac{q_{1}^{n}}{p_{1}^{n}} b+\left(1-\frac{q_{1}^{n}}{p_{1}^{n}}\right) a, \frac{q_{2}^{m}}{p_{2}^{m}} d+\left(1-\frac{q_{2}^{m}}{p_{2}^{m}}\right) c\right) \\
& =-\frac{h(b, c)}{(b-a)(d-c)}-\frac{1}{(b-a)(d-c)} \sum_{n=1}^{\infty} \sum_{m=1}^{\infty} h\left(\frac{q_{1}^{n}}{p_{1}^{n}} b+\left(1-\frac{q_{1}^{n}}{p_{1}^{n}}\right) a, \frac{q_{2}^{m}}{p_{2}^{m}} d+\left(1-\frac{q_{2}^{m}}{p_{2}^{m}}\right) c\right) \text {, } \\
& \frac{1}{(b-a)(d-c)} \sum_{n=0}^{\infty} \sum_{m=0}^{\infty} h\left(\frac{q_{1}^{n}}{p_{1}^{n}} b+\left(1-\frac{q_{1}^{n}}{p_{1}^{n}}\right) a, \frac{q_{2}^{m}}{p_{2}^{m}} d+\left(1-\frac{q_{2}^{m}}{p_{2}^{m}}\right) c\right) \\
& =\frac{h(b, d)}{(b-a)(d-c)}+\frac{1}{(b-a)(d-c)} \sum_{n=1}^{\infty} \sum_{m=1}^{\infty} h\left(\frac{q_{1}^{n}}{p_{1}^{n}} b+\left(1-\frac{q_{1}^{n}}{p_{1}^{n}}\right) a, \frac{q_{2}^{m}}{p_{2}^{m}} d+\left(1-\frac{q_{2}^{m}}{p_{2}^{m}}\right) c\right) \text {, } \\
& -\frac{\left(p_{1}+q_{1}\right)}{q_{1}(b-a)(d-c)} \sum_{n=1}^{\infty} \sum_{m=1}^{\infty} \frac{q_{1}^{n}}{p_{1}^{n}} h\left(\frac{q_{1}^{n}}{p_{1}^{n}} b+\left(1-\frac{q_{1}^{n}}{p_{1}^{n}}\right) a, \frac{q_{2}^{m}}{p_{2}^{m}} d+\left(1-\frac{q_{2}^{m}}{p_{2}^{m}}\right) c\right) \\
& =\frac{\left(p_{1}+q_{1}\right) h(b, c)}{q_{1}(b-a)(d-c)}-\frac{\left(p_{1}+q_{1}\right)}{q_{1}(b-a)(d-c)} \sum_{n=0}^{\infty} \frac{q_{1}^{n}}{p_{1}^{n}} h\left(\frac{q_{1}^{n}}{p_{1}^{n}} b+\left(1-\frac{q_{1}^{n}}{p_{1}^{n}}\right) a, c\right) \\
& -\frac{\left(p_{1}+q_{1}\right)}{q_{1}(b-a)(d-c)} \sum_{n=1}^{\infty} \sum_{m=1}^{\infty} \frac{q_{1}^{n}}{p_{1}^{n}} h\left(\frac{q_{1}^{n}}{p_{1}^{n}} b+\left(1-\frac{q_{1}^{n}}{p_{1}^{n}}\right) a, \frac{q_{2}^{m}}{p_{2}^{m}} d+\left(1-\frac{q_{2}^{m}}{p_{2}^{m}}\right) c\right) \text {, } \\
& \frac{\left(p_{1}+q_{1}\right)}{q_{1}(b-a)(d-c)} \sum_{n=1}^{\infty} \sum_{m=0}^{\infty} \frac{q_{1}^{n}}{p_{1}^{n}} h\left(\frac{q_{1}^{n}}{p_{1}^{n}} b+\left(1-\frac{q_{1}^{n}}{p_{1}^{n}}\right) a, \frac{q_{2}^{m}}{p_{2}^{m}} d+\left(1-\frac{q_{2}^{m}}{p_{2}^{m}}\right) c\right) \\
& =-\frac{\left(p_{1}+q_{1}\right) h(b, d)}{q_{1}(b-a)(d-c)}+\frac{\left(p_{1}+q_{1}\right)}{q_{1}(b-a)(d-c)} \sum_{n=0}^{\infty} \frac{q_{1}^{n}}{p_{1}^{n}} h\left(\frac{q_{1}^{n}}{p_{1}^{n}} b+\left(1-\frac{q_{1}^{n}}{p_{1}^{n}}\right) a, d\right) \\
& +\frac{\left(p_{1}+q_{1}\right)}{q_{1}(b-a)(d-c)} \sum_{n=1}^{\infty} \sum_{m=1}^{\infty} \frac{q_{1}^{n}}{p_{1}^{n}} h\left(\frac{q_{1}^{n}}{p_{1}^{n}} b+\left(1-\frac{q_{1}^{n}}{p_{1}^{n}}\right) a, \frac{q_{2}^{m}}{p_{2}^{m}} d+\left(1-\frac{q_{2}^{m}}{p_{2}^{m}}\right) c\right), \\
& \frac{\left(p_{1}+q_{1}\right)}{p_{1}(b-a)(d-c)} \sum_{n=0}^{\infty} \sum_{m=1}^{\infty} \frac{q_{1}^{n}}{p_{1}^{n}} h\left(\frac{q_{1}^{n}}{p_{1}^{n}} b+\left(1-\frac{q_{1}^{n}}{p_{1}^{n}}\right) a, \frac{q_{2}^{m}}{p_{2}^{m}} d+\left(1-\frac{q_{2}^{m}}{p_{2}^{m}}\right) c\right) \\
& =\frac{\left(p_{1}+q_{1}\right)}{p_{1}(b-a)(d-c)}\left[-\sum_{n=0}^{\infty} \frac{q_{1}^{n}}{p_{1}^{n}} h\left(\frac{q_{1}^{n}}{p_{1}^{n}} b+\left(1-\frac{q_{1}^{n}}{p_{1}^{n}}\right) a, d\right)\right. \\
& \left.+\sum_{n=0}^{\infty} \frac{q_{1}^{n}}{p_{1}^{n}} h\left(\frac{q_{1}^{n}}{p_{1}^{n}} b+\left(-\frac{q_{1}^{n}}{p_{1}^{n}}\right) a, c\right)\right]+\frac{\left(p_{1}+q_{1}\right)}{p_{1}(b-a)(d-c)} \\
& \times \sum_{n=0}^{\infty} \sum_{m=0}^{\infty} \frac{q_{1}^{n}}{p_{1}^{n}} h\left(\frac{q_{1}^{n}}{p_{1}^{n}} b+\left(1-\frac{q_{1}^{n}}{p_{1}^{n}}\right) a, \frac{q_{2}^{m}}{p_{2}^{m}} d+\left(1-\frac{q_{2}^{m}}{p_{2}^{m}}\right) c\right), \\
& -\frac{\left(p_{2}+q_{2}\right)}{q_{2}(b-a)(d-c)} \sum_{n=1}^{\infty} \sum_{m=1}^{\infty} \frac{q_{2}^{m}}{p_{2}^{m}} h\left(\frac{q_{1}^{n}}{p_{1}^{n}} b+\left(1-\frac{q_{1}^{n}}{p_{1}^{n}}\right) a, \frac{q_{2}^{m}}{p_{2}^{m}} d+\left(1-\frac{q_{2}^{m}}{p_{2}^{m}}\right) c\right) \\
& =\frac{\left(p_{2}+q_{2}\right) h(a, d)}{q_{2}(b-a)(d-c)}-\frac{\left(p_{2}+q_{2}\right)}{q_{2}(b-a)(d-c)} \sum_{m=0}^{\infty} \frac{q_{2}^{m}}{p_{2}^{m}} h\left(a, \frac{q_{2}^{m}}{p_{2}^{m}} d+\left(1-\frac{q_{2}^{m}}{p_{2}^{m}}\right) c\right) \\
& -\frac{\left(p_{2}+q_{2}\right)}{q_{2}(b-a)(d-c)} \sum_{n=1}^{\infty} \sum_{m=1}^{\infty} \frac{q_{2}^{m}}{p_{2}^{m}} h\left(\frac{q_{1}^{n}}{p_{1}^{n}} b+\left(1-\frac{q_{1}^{n}}{p_{1}^{n}}\right) a, \frac{q_{2}^{m}}{p_{2}^{m}} d+\left(1-\frac{q_{2}^{m}}{p_{2}^{m}}\right) c\right) \text {, }
\end{aligned}
$$




$$
\begin{aligned}
& \frac{\left(p_{2}+q_{2}\right)}{p_{2}(b-a)(d-c)} \sum_{n=1}^{\infty} \sum_{m=0}^{\infty} \frac{q_{2}^{m}}{p_{2}^{m}} h\left(\frac{q_{1}^{n}}{p_{1}^{n}} b+\left(1-\frac{q_{1}^{n}}{p_{1}^{n}}\right) a, \frac{q_{2}^{m}}{p_{2}^{m}} d+\left(1-\frac{q_{2}^{m}}{p_{2}^{m}}\right) c\right) \\
& =-\frac{\left(p_{2}+q_{2}\right)}{p_{2}(b-a)(d-c)}\left[-\sum_{m=0}^{\infty} \frac{q_{2}^{m}}{p_{2}^{m}} h\left(b, \frac{q_{2}^{m}}{p_{2}^{m}} d+\left(1-\frac{q_{2}^{m}}{p_{2}^{m}}\right) c\right)\right. \\
& \left.+\sum_{m=0}^{\infty} \frac{q_{2}^{m}}{p_{2}^{m}} h\left(a, \frac{q_{2}^{m}}{p_{2}^{m}} d+\left(1-\frac{q_{2}^{m}}{p_{2}^{m}}\right) c\right)\right]+\frac{\left(p_{2}+q_{2}\right)}{p_{2}(b-a)(d-c)} \\
& \times \sum_{n=0}^{\infty} \sum_{m=0}^{\infty} \frac{q_{2}^{m}}{p_{2}^{m}} h\left(\frac{q_{1}^{n}}{p_{1}^{n}} b+\left(1-\frac{q_{1}^{n}}{p_{1}^{n}}\right) a, \frac{q_{2}^{m}}{p_{2}^{m}} d+\left(1-\frac{q_{2}^{m}}{p_{2}^{m}}\right) c\right), \\
& \frac{\left(p_{2}+q_{2}\right)}{q_{2}(b-a)(d-c)} \sum_{n=0}^{\infty} \sum_{m=1}^{\infty} \frac{q_{2}^{m}}{p_{2}^{m}} h\left(\frac{q_{1}^{n}}{p_{1}^{n}} b+\left(1-\frac{q_{1}^{n}}{p_{1}^{n}}\right) a, \frac{q_{2}^{m}}{p_{2}^{m}} d+\left(1-\frac{q_{2}^{m}}{p_{2}^{m}}\right) c\right) \\
& =-\frac{\left(p_{2}+q_{2}\right) h(b, d)}{q_{2}(b-a)(d-c)}+\frac{\left(p_{2}+q_{2}\right)}{q_{2}(b-a)(d-c)} \sum_{m=0}^{\infty} \frac{q_{2}^{m}}{p_{2}^{m}} h\left(b, \frac{q_{2}^{m}}{p_{2}^{m}} d+\left(1-\frac{q_{2}^{m}}{p_{2}^{m}}\right) c\right) \\
& +\frac{\left(p_{2}+q_{2}\right)}{q_{2}(b-a)(d-c)} \sum_{n=1}^{\infty} \sum_{m=1}^{\infty} \frac{q_{2}^{m}}{p_{2}^{m}} h\left(\frac{q_{1}^{n}}{p_{1}^{n}} b+\left(1-\frac{q_{1}^{n}}{p_{1}^{n}}\right) a, \frac{q_{2}^{m}}{p_{2}^{m}} d+\left(1-\frac{q_{2}^{m}}{p_{2}^{m}}\right) c\right) \text {, } \\
& \frac{\left(p_{1}+q_{1}\right)\left(p_{2}+q_{2}\right)}{q_{1} q_{2}(b-a)(d-c)} \sum_{n=1}^{\infty} \sum_{m=1}^{\infty} \frac{q_{1}^{n}}{p_{1}^{n}} \frac{q_{2}^{m}}{p_{2}^{m}} h\left(\frac{q_{1}^{n}}{p_{1}^{n}} b+\left(1-\frac{q_{1}^{n}}{p_{1}^{n}}\right) a, \frac{q_{2}^{m}}{p_{2}^{m}} d+\left(1-\frac{q_{2}^{m}}{p_{2}^{m}}\right) c\right) \\
& =-\frac{\left(p_{1}+q_{1}\right)\left(p_{2}+q_{2}\right) h(b, d)}{q_{1} q_{2}(b-a)(d-c)}-\frac{\left(p_{1}+q_{1}\right)\left(p_{2}+q_{2}\right)}{q_{1} q_{2}(b-a)(d-c)} \sum_{n=0}^{\infty} \frac{q_{1}^{n}}{p_{1}^{n}} h\left(\frac{q_{1}^{n}}{p_{1}^{n}} b+\left(1-\frac{q_{1}^{n}}{p_{1}^{n}}\right) a, d\right) \\
& -\frac{\left(p_{1}+q_{1}\right)\left(p_{2}+q_{2}\right)}{q_{1} q_{2}(b-a)(d-c)} \sum_{m=0}^{\infty} \frac{q_{2}^{m}}{p_{2}^{m}} h\left(b, \frac{q_{2}^{m}}{p_{2}^{m}} d+\left(1-\frac{q_{2}^{m}}{p_{2}^{m}}\right) c\right) \\
& +\frac{\left(p_{1}+q_{1}\right)\left(p_{2}+q_{2}\right)}{q_{1} q_{2}(b-a)(d-c)} \sum_{n=0}^{\infty} \sum_{m=0}^{\infty} \frac{q_{1}^{n}}{p_{1}^{n}} \frac{q_{2}^{m}}{p_{2}^{m}} h\left(\frac{q_{1}^{n}}{p_{1}^{n}} b+\left(1-\frac{q_{1}^{n}}{p_{1}^{n}}\right) a, \frac{q_{2}^{m}}{p_{2}^{m}} d+\left(1-\frac{q_{2}^{m}}{p_{2}^{m}}\right) c\right), \\
& -\frac{\left(p_{1}+q_{1}\right)\left(p_{2}+q_{2}\right)}{p_{2} q_{1}(b-a)(d-c)} \sum_{n=1}^{\infty} \sum_{m=0}^{\infty} \frac{q_{1}^{n}}{p_{1}^{n}} \frac{q_{2}^{m}}{p_{2}^{m}} h\left(\frac{q_{1}^{n}}{p_{1}^{n}} b+\left(1-\frac{q_{1}^{n}}{p_{1}^{n}}\right) a, \frac{q_{2}^{m}}{p_{2}^{m}} d+\left(1-\frac{q_{2}^{m}}{p_{2}^{m}}\right) c\right) \\
& =\frac{\left(p_{1}+q_{1}\right)\left(p_{2}+q_{2}\right)}{p_{2} q_{1}(b-a)(d-c)} \sum_{m=0}^{\infty} \frac{q_{2}^{m}}{p_{2}^{m}} h\left(b, \frac{q_{2}^{m}}{p_{2}^{m}} d+\left(1-\frac{q_{2}^{m}}{p_{2}^{m}}\right) c\right) \\
& -\frac{\left(p_{1}+q_{1}\right)\left(p_{2}+q_{2}\right)}{p_{2} q_{1}(b-a)(d-c)} \sum_{n=0}^{\infty} \sum_{m=0}^{\infty} \frac{q_{1}^{n}}{p_{1}^{n}} \frac{q_{2}^{m}}{p_{2}^{m}} h\left(\frac{q_{1}^{n}}{p_{1}^{n}} b+\left(\left(1-\frac{q_{1}^{n}}{p_{1}^{n}}\right) a, \frac{q_{2}^{m}}{p_{2}^{m}} d+\left(\left(1-\frac{q_{2}^{m}}{p_{2}^{m}}\right) c\right)\right. \text {, }\right. \\
& -\frac{\left(p_{1}+q_{1}\right)\left(p_{2}+q_{2}\right)}{p_{1} q_{2}(b-a)(d-c)} \sum_{n=0}^{\infty} \sum_{m=1}^{\infty} \frac{q_{1}^{n}}{p_{1}^{n}} \frac{q_{2}^{m}}{p_{2}^{m}} h\left(\frac{q_{1}^{n}}{p_{1}^{n}} b+\left(1-\frac{q_{1}^{n}}{p_{1}^{n}}\right) a, \frac{q_{2}^{m}}{p_{2}^{m}} d+\left(1-\frac{q_{2}^{m}}{p_{2}^{m}}\right) c\right) \\
& =\frac{\left(p_{1}+q_{1}\right)\left(p_{2}+q_{2}\right)}{p_{1} q_{2}(b-a)(d-c)} \sum_{n=0}^{\infty} \frac{q_{1}^{n}}{p_{1}^{n}} h\left(\frac{q_{1}^{n}}{p_{1}^{n}} b+\left(1-\frac{q_{1}^{n}}{p_{1}^{n}}\right) a, d\right) \\
& -\frac{\left(p_{1}+q_{1}\right)\left(p_{2}+q_{2}\right)}{p_{1} q_{2}(b-a)(d-c)} \sum_{n=0}^{\infty} \sum_{m=0}^{\infty} \frac{q_{1}^{n}}{p_{1}^{n}} \frac{q_{2}^{m}}{p_{2}^{m}} h\left(\frac{q_{1}^{n}}{p_{1}^{n}} b+\left(1-\frac{q_{1}^{n}}{p_{1}^{n}}\right) a, \frac{q_{2}^{m}}{p_{2}^{m}} d+\left(1-\frac{q_{2}^{m}}{p_{2}^{m}}\right) c\right) \text {. }
\end{aligned}
$$

Using (20)-(32) and simplifying, we get:

$$
\begin{aligned}
& \int_{0}^{1} \int_{0}^{1}\left(1-\left(p_{1}+q_{1}\right) z\right)\left(1-\left(p_{2}+q_{2}\right) w\right) \frac{a_{,} \partial_{p_{1} p_{2}, q_{1} q_{2}}^{2} h((1-z) a+z b,(1-w) c+w d)}{{ }_{a} \partial_{p_{1}, q_{1}} z_{c} \partial_{p_{1}, q_{1}} w}{ }_{0} d_{p_{1}, q_{1}} z_{0} d_{p_{2}, q_{2}} w \\
& =\frac{q_{1} q_{2} h(a, c)+p_{2} q_{1} h(a, d)+p_{1} q_{2} h(b, c)+p_{1} p_{2} h(b, d)}{q_{1} q_{2}(b-a)(d-c)}
\end{aligned}
$$




$$
\begin{aligned}
& -\frac{\left(p_{1}+q_{1}\right)\left(p_{1}-q_{1}\right)}{p_{1} q_{1}(b-a)(d-c)} \sum_{n=0}^{\infty} \frac{q_{1}^{n}}{p_{1}^{n}} h\left(\frac{q_{1}^{n}}{p_{1}^{n}} b+\left(1-\frac{q_{1}^{n}}{p_{1}^{n}}\right) a, c\right) \\
& -\frac{p_{2}\left(p_{1}+q_{1}\right)\left(p_{1}-q_{1}\right)}{p_{1} q_{1} q_{2}(b-a)(d-c)} \sum_{n=0}^{\infty} \frac{q_{1}^{n}}{p_{1}^{n}} h\left(\frac{q_{1}^{n}}{p_{1}^{n}} b+\left(1-\frac{q_{1}^{n}}{p_{1}^{n}}\right) a, d\right) \\
& -\frac{\left(p_{2}+q_{2}\right)\left(p_{2}-q_{2}\right)}{p_{2} q_{2}(b-a)(d-c)} \sum_{m=0}^{\infty} \frac{q_{2}^{m}}{p_{2}^{m}} h\left(a, \frac{q_{2}^{m}}{p_{2}^{m}} d+\left(1-\frac{q_{2}^{m}}{p_{2}^{m}}\right) c\right) \\
& -\frac{p_{1}\left(p_{2}+q_{2}\right)\left(p_{2}-q_{2}\right)}{p_{2} q_{1} q_{2}(b-a)(d-c)} \sum_{m=0}^{\infty} \frac{q_{2}^{m}}{p_{2}^{m}} h\left(b, \frac{q_{2}^{m}}{p_{2}^{m}} d+\left(1-\frac{q_{2}^{m}}{p_{2}^{m}}\right) c\right) \\
& +\frac{\left(p_{1}+q_{1}\right)\left(p_{2}+q_{2}\right)\left(p_{1}-q_{1}\right)\left(p_{2}-q_{2}\right)}{p_{1} p_{2} q_{1} q_{2}(b-a)(d-c)} \\
& \times \sum_{n=0}^{\infty} \sum_{m=0}^{\infty} \frac{q_{1}^{n}}{p_{1}^{n}} \frac{q_{2}^{m}}{p_{2}^{m}} h\left(\frac{q_{1}^{n}}{p_{1}^{n}} b+\left(1-\frac{q_{1}^{n}}{p_{1}^{n}}\right) a, \frac{q_{2}^{m}}{p_{2}^{m}} d+\left(1-\frac{q_{2}^{m}}{p_{2}^{m}}\right) c\right) \\
& =\frac{q_{1} q_{2} h(a, c)+p_{2} q_{1} h(a, d)+p_{1} q_{2} h(b, c)+p_{1} p_{2} h(b, d)}{q_{1} q_{2}(b-a)(d-c)} \\
& -\frac{\left(p_{1}+q_{1}\right)}{p_{1} q_{1}(b-a)^{2}(d-c)} \int_{a}^{p_{1} b+\left(1-p_{1}\right) a} h(s, c) \quad d_{p_{1}, q_{1} s} \\
& -\frac{p_{2}\left(p_{1}+q_{1}\right)}{p_{1} q_{1} q_{2}(b-a)^{2}(d-c)} \int_{a}^{p_{1} b+\left(1-p_{1}\right) a} h(s, d)_{a} d_{p_{1}, q_{1} s} \\
& -\frac{\left(p_{2}+q_{2}\right)}{p_{2} q_{2}(b-a)(d-c)^{2}} \int_{c}^{p_{2} d+\left(1-p_{2}\right) c} h(a, t)_{c} d_{p_{2}, q_{2}} t \\
& -\frac{p_{1}\left(p_{2}+q_{2}\right)}{p_{2} q_{1} q_{2}(b-a)(d-c)^{2}} \int_{c}^{p_{2} d+\left(1-p_{2}\right) c} h(b, t)_{c} d_{p_{2}, q_{2}} t \\
& +\frac{\left(p_{1}+q_{1}\right)\left(p_{2}+q_{2}\right)}{p_{1} p_{2} q_{1} q_{2}(b-a)^{2}(d-c)^{2}} \int_{a}^{p_{1} b+\left(1-p_{1}\right) a} \int_{c}^{p_{2} d+\left(1-p_{2}\right) c} h(s, t)_{c} d_{p_{2}, q_{2}} t_{a} d_{p_{1}, q_{1}} s . \\
&
\end{aligned}
$$

Multiplying both sides of (33) by $\frac{q_{1} q_{2}(b-a)(d-c)}{\left(p_{1}+q_{1}\right)\left(p_{2}+q_{2}\right)}$, we get the desired equality.

Remark 3. 1. Setting $q_{i} \rightarrow 1^{-}$and $p_{i}=1$ for $i=1,2$ in Lemma 1, then Lemma 1 reduces to Lemma 1 proved in [25].

2. Furthermore, if $p_{i}=1$ for $i=1,2$ in Lemma 1, then Lemma 1 reduces to Lemma 2 proven in [22].

Now, we can introduce quantum integral inequalities for functions whose partial $\left(p_{1} p_{2}, q_{1} q_{2}\right)$-derivative on $\triangle^{0}$ satisfies the convexity on coordinates on $\triangle$.

Theorem 8. Let $h: \triangle \subset \mathbb{R}^{2} \rightarrow \mathbb{R}$ be a function such that ( $\left.p_{1} p_{2}, q_{1} q_{2}\right)$-derivatives exist on $\triangle^{0}$ (the interior of $\triangle$ ) and $0<q_{i}<p_{i} \leq 1$ for $i=1$, 2. Moreover, if $\frac{a, c}{a \partial p_{1}, q_{1} p_{2}, z_{c} \partial_{p_{2}}, q_{2} w}$ is continuous and integrable on $[a, b] \times[c, d] \subset \triangle^{0}$ and $\left.\left|\frac{a, c}{a} \partial_{p_{1}}^{2} p_{2, q_{1} q_{2}} h\right|^{r}{ }_{p_{1}, q_{1}} z_{c} \partial_{p_{2}, q_{2}} w\right|^{r}$ is a convex function on the coordinates on $[a, b] \times[c, d]$ for $r \geq 1$, then the given inequality holds:

$$
\begin{aligned}
& \left|\Gamma_{\left(p_{1} p_{2}, q_{1} q_{2}\right)}(a, b, c, d)(h)\right| \leq \frac{q_{1} q_{2}(b-a)(d-c)}{\left(p_{1}+q_{1}\right)\left(p_{2}+q_{2}\right)}\left(\phi_{p_{1}, q_{1}} \phi_{p_{2}, q_{2}}\right)^{\frac{r-1}{r}} \\
& \times\left(\psi_{p_{1}, q_{1}} \psi_{p_{2}, q_{2}}\left|\frac{a, c \partial_{p_{1} p_{2}, q_{1} q_{2}}^{2} h(a, c)}{a \partial_{p_{1}, q_{1}} z_{c} \partial_{p_{2}, q_{2}} w}\right|^{r}+\psi_{p_{1}, q_{1}} \Delta_{p_{2}, q_{2}}\left|\frac{a, c \partial_{p_{1} p_{2}, q_{1} q_{2}}^{2} h(a, d)}{a \partial_{p_{1}, q_{1}} z_{c} \partial_{p_{2}, q_{2}} w}\right|^{r}\right.
\end{aligned}
$$

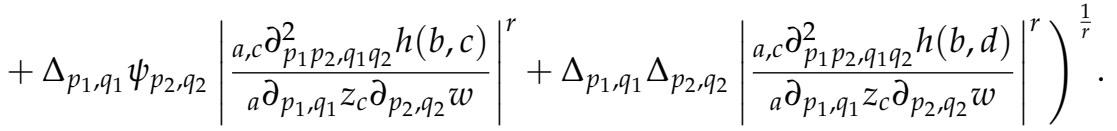


Proof. By applying Lemma 1, using the $\left(p_{1} p_{2}, q_{1} q_{2}\right)$-Hölder inequality, and the convexity of

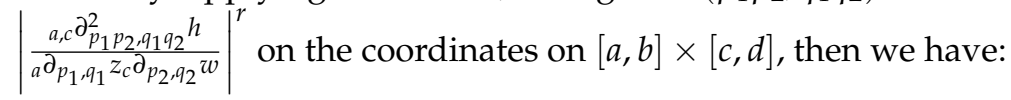

$$
\begin{aligned}
& \left|\Gamma_{\left(p_{1} p_{2}, q_{1} q_{2}\right)}(a, b, c, d)(h)\right| \leq \frac{q_{1} q_{2}(b-a)(d-c)}{\left(p_{1}+q_{1}\right)\left(p_{2}+q_{2}\right)} \\
& \times\left(\int_{0}^{1} \int_{0}^{1}\left|\left(1-\left(p_{1}+q_{1}\right) z\right)\left(1-\left(p_{2}+q_{2}\right) w\right)\right|_{0} d_{p_{1}, q_{1}} z_{0} d_{p_{2}, q_{2}} w\right)^{\frac{r-1}{r}} \\
& \times\left(\int_{0}^{1} \int_{0}^{1}\left|\left(1-\left(p_{1}+q_{1}\right) z\right)\left(1-\left(p_{2}+q_{2}\right) w\right)\right|\right)\left[\left((1-z)(1-w)\left|\frac{a, c \partial_{p_{1} p_{2}, q_{1} q_{2}}^{2} h(a, c)}{{ }_{a} \partial_{p_{1}, q_{1}} z_{c} \partial_{p_{2}, q_{2}} w}\right|^{r}\right.\right.
\end{aligned}
$$

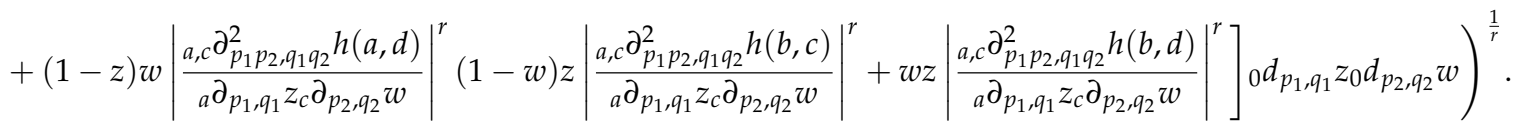

By using Definition 3, we get:

$$
\begin{aligned}
& \phi_{p, q}=\int_{0}^{1}|(1-(p+q) z)|_{0} d_{p, q} z=\frac{2(p+q-1)}{(p+q)^{2}}, \\
& \psi_{p, q}=\int_{0}^{1}(1-z)|(1-(p+q) z)|_{0} d_{p, q} z \\
& =\frac{q\left[\left(p^{3}-2+2 p\right)+\left(2 p^{2}+2\right) q+p q^{2}\right]+2 p^{2}-2 p}{(p+q)^{3}\left(p^{2}+p q+q^{2}\right)}, \\
& \Delta_{p, q}=\int_{0}^{1} z|(1-(p+q) z)|_{0} d_{p, q} z \\
& =\frac{q\left[\left(5 p^{3}-4 p^{2}-2 p+2\right)+\left(6 p^{2}-4 p-2\right) q+(5 p-2) q^{2}+2 q^{3}\right]}{(p+q)^{3}\left(p^{2}+p q+q^{2}\right)} \\
& +\frac{2 p^{4}-2 p^{3}-2 p^{2}+2 p}{(p+q)^{3}\left(p^{2}+p q+q^{2}\right)} .
\end{aligned}
$$

We observe that,

$$
\begin{aligned}
& \int_{0}^{1} \int_{0}^{1}\left|\left(1-\left(p_{1}+q_{1}\right) z\right)\left(1-\left(p_{2}+q_{2}\right) w\right)\right|_{0} d_{p_{1}, q_{1}} z_{0} d_{p_{2}, q_{2}} w \\
& =\left(\int_{0}^{1}\left|\left(1-\left(p_{1}+q_{1}\right) z\right)\right|_{0} d_{p_{1}, q_{1}} z\right)\left(\int_{0}^{1}\left|\left(1-\left(p_{2}+q_{2}\right) w\right)\right|_{0} d_{p_{2}, q_{2}} w\right) \\
& =\phi_{p_{1}, q_{1}} \phi_{p_{2}, q_{2}}, \\
& \left.\int_{0}^{1} \int_{0}^{1}(1-z)(1-w)\left|\left(1-\left(p_{1}+q_{1}\right) z\right)\right|\left|\left(1-\left(p_{2}+q_{2}\right) w\right)\right|_{0} d_{p_{1}, q_{1}} z_{0} d_{p_{2}, q_{2}} w\right) \\
& =\left(\int_{0}^{1}(1-z)\left|\left(1-\left(p_{1}+q_{1}\right) z\right)\right|_{0} d_{p_{1}, q_{1}} z\right)\left(\int_{0}^{1}(1-w)\left|\left(1-\left(p_{2}+q_{2}\right) w\right)\right|_{0} d_{p_{2}, q_{2}} w\right) \\
& =\psi_{p_{1}, q_{1}} \psi_{p_{2}, q_{2}}, \\
& \int_{0}^{1} \int_{0}^{1}(1-z) w\left|\left(1-\left(p_{1}+q_{1}\right) z\right)\left(1-\left(p_{2}+q_{2}\right) w\right)\right|_{0} d_{p_{1}, q_{1}} z_{0} d_{p_{2}, q_{2}} w \\
& =\left(\int_{0}^{1}(1-z)\left|\left(1-\left(p_{1}+q_{1}\right) z\right)\right|_{0} d_{p_{1}, q_{1}} z\right)\left(\int_{0}^{1} w\left|\left(1-\left(p_{2}+q_{2}\right) w\right)\right|_{0} d_{p_{2}, q_{2}} w\right) \\
& =\psi_{p_{1}, q_{1}} \Delta_{p_{2}, q_{2}},
\end{aligned}
$$




$$
\begin{aligned}
& \int_{0}^{1} \int_{0}^{1} z(1-w)\left|\left(1-\left(p_{1}+q_{1}\right) z\right)\left(1-\left(p_{2}+q_{2}\right) w\right)\right|_{0} d_{p_{1}, q_{1}} z_{0} d_{p_{2}, q_{2}} w \\
& =\left(\int_{0}^{1} z\left|\left(1-\left(p_{1}+q_{1}\right) z\right)\right|{ }_{0} d_{p_{1}, q_{1}} z\right)\left(\int_{0}^{1}(1-w)\left|\left(1-\left(p_{2}+q_{2}\right) w\right)\right|_{0} d_{p_{2}, q_{2}} w\right) \\
& =\Delta_{p_{1}, q_{1}} \psi_{p_{2}, q_{2}}, \\
& \int_{0}^{1} \int_{0}^{1} z w\left|\left(1-\left(p_{1}+q_{1}\right) z\right)\left(1-\left(p_{2}+q_{2}\right) w\right)\right|_{0} d_{p_{1}, q_{1}} z_{0} d_{p_{2}, q_{2}} w \\
& =\left(\int_{0}^{1} z\left|\left(1-\left(p_{1}+q_{1}\right) z\right)\right|_{0} d_{p_{1}, q_{1}} z\right)\left(\int_{0}^{1} w\left|\left(1-\left(p_{2}+q_{2}\right) w\right)\right|_{0} d_{p_{2}, q_{2}} w\right) \\
& =\Delta_{p_{1}, q_{1}} \Delta_{p_{2}, q_{2}} .
\end{aligned}
$$

Using the values of the above $\left(p_{1} p_{2}, q_{1} q_{2}\right)$-integrals, we get the desired inequality.

Theorem 9. Let $h: \triangle \subset \mathbb{R}^{2} \rightarrow \mathbb{R}$ be a function such that ( $\left.p_{1} p_{2}, q_{1} q_{2}\right)$-derivatives exist on $\triangle^{\mathrm{o}}$ (the interior of

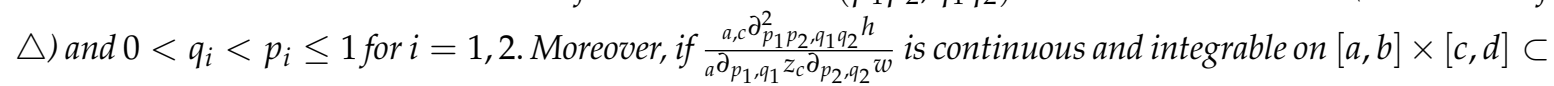
$\triangle^{\mathrm{o}}$ and $\left.\left|\frac{a, c}{a_{p_{1}, q_{1}} z_{2}, p_{p_{2}, q_{2}} h}\right|^{r_{1}}\right|^{r_{1}}$ is a convex function on the coordinates on $[a, b] \times[c, d]$. If $r_{1}^{-1}+r_{2}^{-1}=1$ and $r_{1}>1$, then the given inequality holds:

$$
\begin{aligned}
& \left|\Gamma_{\left(p_{1} p_{2}, q_{1} q_{2}\right)}(a, b, c, d)(h)\right| \leq \frac{q_{1} q_{2}(b-a)(d-c)}{\left(\left(p_{1}+q_{1}\right)\left(p_{2}+q_{2}\right)\right)^{1+\frac{1}{r_{1}}}}\left(B_{p_{1}, q_{1}}\left(r_{2}\right) B_{p_{2}, q_{2}}\left(r_{2}\right)\right)^{\frac{1}{r_{2}}}
\end{aligned}
$$

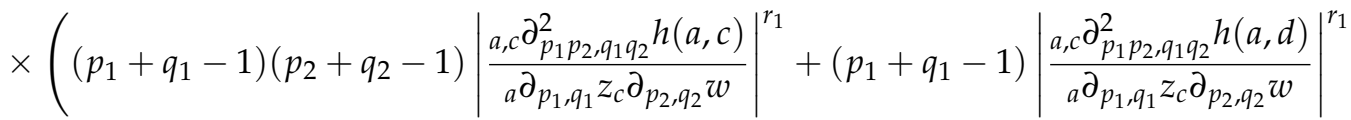

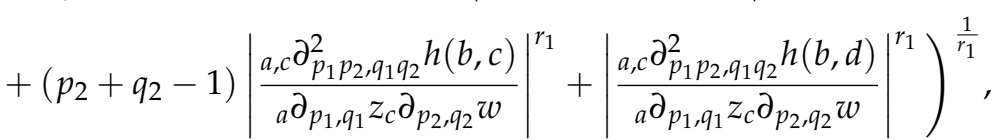

Proof. By applying Lemma 1, the $\left(p_{1} p_{2}, q_{1} q_{2}\right)$-Hölder inequality, and the convexity of $\left|\frac{a, c}{a \partial_{p_{1}, q_{1}} z_{c} p_{c}, q_{2}, q_{2}, q_{2}}\right|^{r_{1} w}$ on the coordinates on $[a, b] \times[c, d]$, then we have:

$$
\begin{aligned}
& \left|\Gamma_{\left(p_{1} p_{2}, q_{1} q_{2}\right)}(a, b, c, d)(h)\right| \leq \frac{q_{1} q_{2}(b-a)(d-c)}{\left(p_{1}+q_{1}\right)\left(p_{2}+q_{2}\right)} \\
& \times\left(\int_{0}^{1} \int_{0}^{1}\left|\left(1-\left(p_{1}+q_{1}\right) z\right)\left(1-\left(p_{2}+q_{2}\right) w\right)\right|^{r_{2}}{ }_{0} d_{p_{1}, q_{1}} z_{0} d_{p_{2}, q_{2}} w\right)^{\frac{1}{r_{2}}} \\
& \times\left(\int _ { 0 } ^ { 1 } \int _ { 0 } ^ { 1 } \left[(1-z)(1-w)\left|\frac{a, c}{{ }_{a} \partial_{p_{1}, p_{2}, q_{1} q_{2}}^{2} z_{c} \partial_{p_{2}, q_{2}} w}\right|^{2}+(1-z) w\left|\frac{a, c \partial_{p_{1} p_{2}, q_{1} q_{2}}^{2} h(a, d)}{{ }_{a} \partial_{p_{1}, q_{1}} z_{c} \partial_{p_{2}, q_{2}} w}\right|^{r_{1}}\right.\right. \\
& \left.\left.+(1-w) z\left|\frac{a, c}{{ }_{a} \partial_{p_{1}, q_{1}, q_{1} q_{2}}^{2} z_{p_{2}, q_{2}} w}\right|^{r_{1}}+w z\left|\frac{a, c \partial_{p_{1} p_{2}, q_{1} q_{2}}^{2} h(b, d)}{{ }_{a} \partial_{p_{1}, q_{1}} z_{c} \partial p_{2}, q_{2}}\right|^{r_{1}}\right]{ }_{0} d_{p_{1}, q_{1}} z_{0} d_{p_{2}, q_{2}} w\right)^{\frac{1}{r_{1}}} .
\end{aligned}
$$

We calculate as,

$$
\int_{0}^{1}\left|\left(1-\left(p_{1}+q_{1}\right) z\right)\right|^{r_{2}}{ }_{0} d_{p_{1}, q_{1}} z=\int_{0}^{\frac{1}{p_{1}+q_{1}}}\left(1-\left(p_{1}+q_{1}\right) z\right)^{r_{2}} 0 d_{p_{1}, q_{1}} z+\int_{\frac{1}{p_{1}+q_{1}}}^{1}\left(\left(p_{1}+q_{1}\right) z-1\right)^{r_{2}} d_{p_{1}, q_{1}} z .
$$


Considering the first $\left(p_{1}, q_{1}\right)$-integral from (34) and making use of the substitution $1-\left(p_{1}+q_{1}\right) z=$ $w$, we obtain:

$$
\int_{0}^{\frac{1}{p_{1}+q_{1}}}\left(1-\left(p_{1}+q_{1}\right) z\right)^{r_{2}} d_{p_{1}, q_{1}} z=\frac{1}{p_{1}+q_{1}} \int_{0}^{1} w^{r_{2}}{ }_{0} d_{p_{1}, q_{1}} w=\frac{p_{1}-q_{1}}{\left(p_{1}+q_{1}\right)\left(p_{1}^{r_{2}+1}-q_{1}^{r_{2}+1}\right)} .
$$

Considering the second $\left(p_{1}, q_{1}\right)$-integral from (34) and making use of the substitution $\left(p_{1}+q_{1}\right) z-$ $1=w$, we obtain:

$$
\begin{gathered}
\int_{\frac{1}{p_{1}+q_{1}}}^{1}\left(\left(p_{1}+q_{1}\right) z-1\right)^{r_{2}} d_{p_{1}, q_{1}} z=\frac{1}{p_{1}+q_{1}} \int_{0}^{p_{1}+q_{1}-1} w^{r_{2}} d_{p_{1}, q_{1}} w \\
=\frac{\left(p_{1}-q_{1}\right)\left(p_{1}+q_{1}-1\right)^{r_{2}+1}}{\left(p_{1}+q_{1}\right)\left(p_{1}^{r_{2}+1}-q_{1}^{r_{2}+1}\right)} .
\end{gathered}
$$

After this calculation, we get:

$$
\int_{0}^{1}\left|\left(1-\left(p_{1}+q_{1}\right) z\right)\right|^{r_{2}}{ }_{0} d_{p_{1}, q_{1}} z=\frac{\left(p_{1}-q_{1}\right)\left(1+\left(p_{1}+q_{1}-1\right)^{r_{2}+1}\right)}{\left(p_{1}+q_{1}\right)\left(p_{1}^{r_{2}+1}-q_{1}^{r_{2}+1}\right)}=B_{p_{1}, q_{1}}\left(r_{2}\right) .
$$

Similarly, one can have:

$$
\int_{0}^{1}\left|\left(1-\left(p_{2}+q_{2}\right) w\right)\right|^{r_{2}}{ }_{0} d_{p_{2}, q_{2}} w=\frac{\left(p_{2}-q_{2}\right)\left(1+\left(p_{2}+q_{2}-1\right)^{r_{2}+1}\right)}{\left(p_{2}+q_{2}\right)\left(p_{2}^{r_{2}+1}-q_{2}^{r_{2}+1}\right)}=B_{p_{2}, q_{2}}\left(r_{2}\right) .
$$

Finally, we also have:

$$
\begin{aligned}
& \int_{0}^{1}(1-z)_{0} d_{p_{1}, q_{1}} z=\frac{p_{1}+q_{1}-1}{p_{1}+q_{1}}, \int_{0}^{1}(1-w)_{0} d_{p_{2}, q_{2}} w=\frac{p_{2}+q_{2}-1}{p_{2}+q_{2}}, \int_{0}^{1} z_{0} d_{p_{1}, q_{1}} z=\frac{1}{p_{1}+q_{1}}, \\
& \int_{0}^{1} w_{0} d_{p_{2}, q_{2}} w=\frac{1}{p_{2}+q_{2}} .
\end{aligned}
$$

Remark 4. 1. Setting $q_{i} \rightarrow 1^{-}$and $p_{i}=1$ for $i=1,2$ in Theorems 8 and 9 , then Theorems 8 and 9 reduce to Theorems 4 and 5 proven in [25].

2. Furthermore, if $p_{i}=1$ for $i=1,2$ in Theorems 8 and 9 , then Theorems 8 and 9 reduce to Theorems 6 and 7 proven in [22].

Theorem 10. Let $h: \triangle \subset \mathbb{R}^{2} \rightarrow \mathbb{R}$ be a function such that $\left(p_{1} p_{2}, q_{1} q_{2}\right)$-derivatives exist on $\triangle^{\mathrm{o}}$ (the interior of $\triangle$ ) and $0<q_{i}<p_{i} \leq 1$ for $i=1,2$. Moreover, if $\frac{a, c \partial \partial_{1}^{2} p_{2}, q_{1} q_{2} h}{a \partial p_{1}, q_{1} z_{c} \partial p_{2}, q_{2} w}$ is continuous and integrable on $[a, b] \times[c, d] \subset \triangle^{\mathrm{o}}$ and $\left|\frac{a, c \partial_{p_{1}}^{2} p_{2}, q_{1} q_{2} h}{a \partial \partial_{1}, q_{1} z_{c} \partial \partial_{2}, q_{2}}\right|^{r}$ is quasi-convex on the coordinates on $[a, b] \times[c, d]$ for $r \geq 1$, then the inequality holds:

$$
\begin{aligned}
& \left|\Gamma_{\left(p_{1} p_{2}, q_{1} q_{2}\right)}(a, b, c, d)(h)\right| \leq \frac{q_{1} q_{2}(b-a)(d-c)}{\left(p_{1}+q_{1}\right)\left(p_{2}+q_{2}\right)}\left(\phi_{p_{1}, q_{1}} \phi_{p_{2}, q_{2}}\right)
\end{aligned}
$$

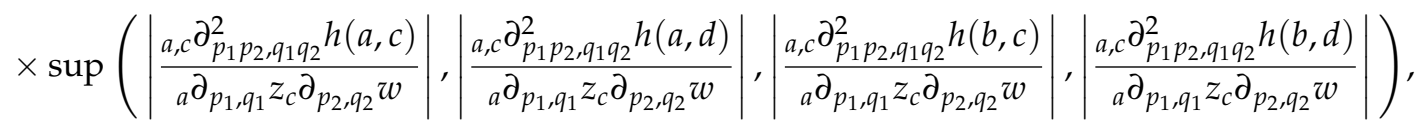

where $\phi_{p_{1}, q_{1}}$ and $\phi_{p_{2}, q_{2}}$ are defined in Theorem 8. 
Proof. By applying Lemma 1, using the $\left(p_{1} p_{2}, q_{1} q_{2}\right)$-Hölder inequality, and the quasi-convexity of

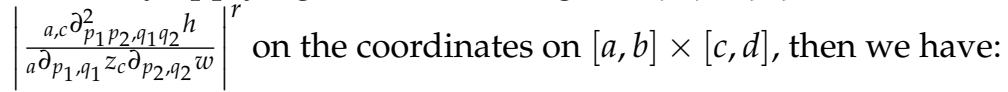

$$
\begin{aligned}
& \left|\Gamma_{\left(p_{1} p_{2}, q_{1} q_{2}\right)}(a, b, c, d)(h)\right| \leq \frac{q_{1} q_{2}(b-a)(d-c)}{\left(p_{1}+q_{1}\right)\left(p_{2}+q_{2}\right)} \\
& \times\left(\int_{0}^{1} \int_{0}^{1}\left|\left(1-\left(p_{1}+q_{1}\right) z\right)\left(1-\left(p_{2}+q_{2}\right) w\right)\right|_{0} d_{p_{1}, q_{1}} z_{0} d_{p_{2}, q_{2}} w\right)^{\frac{r-1}{r}} \\
& \times\left(\int_{0}^{1} \int_{0}^{1}\left|\left(1-\left(p_{1}+q_{1}\right) z\right)\left(1-\left(p_{2}+q_{2}\right) w\right)\right|\right.
\end{aligned}
$$

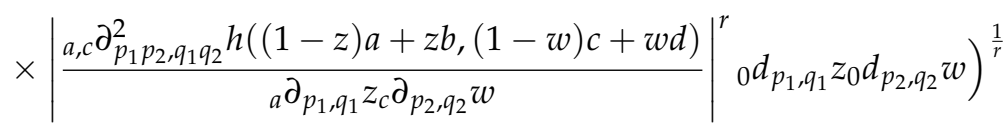

$$
\begin{aligned}
& =\frac{q_{1} q_{2}(b-a)(d-c)}{\left(p_{1}+q_{1}\right)\left(p_{2}+q_{2}\right)}\left(\int_{0}^{1} \int_{0}^{1}\left|\left(1-\left(p_{1}+q_{1}\right) z\right)\left(1-\left(p_{2}+q_{2}\right) w\right)\right|_{0} d_{p_{1}, q_{1}} z_{0} d_{p_{2}, q_{2}} w\right)
\end{aligned}
$$

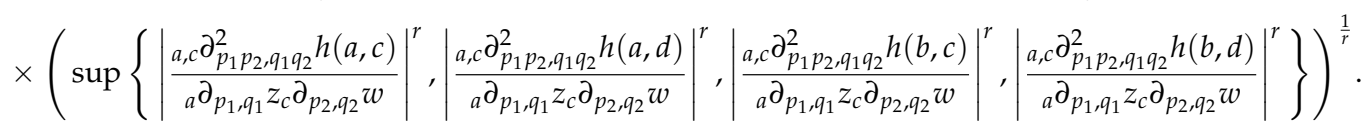

Now, using the properties of the supremum and Definition 3, we get the desired result from (35).

Theorem 11. Let $h: \triangle \subset \mathbb{R}^{2} \rightarrow \mathbb{R}$ be a function such that $\left(p_{1} p_{2}, q_{1} q_{2}\right)$-derivatives exist on $\triangle^{\mathrm{o}}$ (the interior of $\triangle$ ) and $0<q_{i}<p_{i} \leq 1$, for $i=1$, 2. Moreover, if $\frac{a, c}{a \partial p_{1}, q_{1} p_{2}, q_{1} q_{2} q_{2} h}$ 的, continuous and integrable on $[a, b] \times[c, d]$

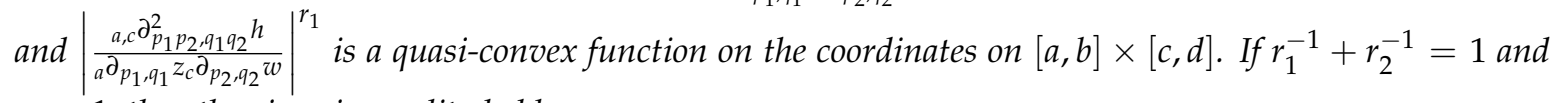
$r_{1}>1$, then the given inequality holds:

$$
\begin{aligned}
& \left|\Gamma_{\left(p_{1} p_{2}, q_{1} q_{2}\right)}(a, b, c, d)(h)\right| \leq \frac{q_{1} q_{2}(b-a)(d-c)}{\left(p_{1}+q_{1}\right)\left(p_{2}+q_{2}\right)}\left(B_{p_{1}, q_{1}}\left(r_{2}\right) B_{p_{2}, q_{2}}\left(r_{2}\right)\right)^{\frac{1}{r_{2}}} \\
& \times \sup \left\{\left|\frac{a, c \partial_{p_{1} p_{2}, q_{1} q_{2}}^{2} h(a, c)}{a \partial_{p_{1}, q_{1}} z_{c} \partial_{p_{2}, q_{2}} w}\right|,\left|\frac{a, c \partial_{p_{1} p_{2}, q_{1} q_{2}}^{2} h(a, d)}{a \partial_{p_{1}, q_{1}} z_{c} \partial_{p_{2}, q_{2}} w}\right|,\left|\frac{a, c \partial_{p_{1}}^{2} p_{2}, q_{1} q_{2}}{\partial_{p_{1}, q_{1}} z_{c} \partial_{p_{2}, q_{2}} w}\right|,\left|\frac{a, c \partial_{p_{1}}^{2} p_{2}, q_{1} q_{2}}{\partial_{p_{1}, q_{1}} z_{c} \partial_{p_{2}, q_{2}} w}\right|\right\},
\end{aligned}
$$

where $B_{p_{1}, q_{1}}\left(r_{2}\right)$ and $B_{p_{2}, q_{2}}\left(r_{2}\right)$ are defined in Theorem 9.

Proof. By applying Lemma 1, using the $\left(p_{1} p_{2}, q_{1} q_{2}\right)$ - Hölder inequality, and the quasi-convexity of

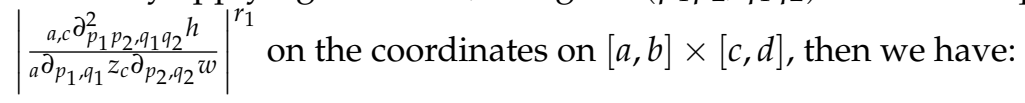

$$
\begin{aligned}
& \left|\Gamma_{\left(p_{1} p_{2}, q_{1} q_{2}\right)}(a, b, c, d)(h)\right| \leq \frac{q_{1} q_{2}(b-a)(d-c)}{\left(p_{1}+q_{1}\right)\left(p_{2}+q_{2}\right)} \\
& \times\left(\int_{0}^{1} \int_{0}^{1}\left|\left(1-\left(p_{1}+q_{1}\right) z\right)\left(1-\left(p_{2}+q_{2}\right) w\right)\right|_{1}^{r} d_{p_{1}, q_{1}} z_{0} d_{p_{2}, q_{2}} w\right)^{\frac{1}{r_{1}}} \\
& \times\left(\int_{0}^{1} \int_{0}^{1}\left|\frac{a, c \partial_{p_{1} p_{2}, q_{1} q_{2}}^{2} h((1-z) a+z b,(1-w) c+w d)}{{ }_{a} \partial_{p_{1}, q_{1}} z_{c} \partial_{p_{2}, q_{2}} w}\right|^{r_{2}}{ }_{0} d_{p_{1}, q_{1}} z_{0} d_{p_{2}, q_{2}} w\right)^{\frac{1}{r_{2}}}
\end{aligned}
$$




$$
\begin{aligned}
& =\frac{q_{1} q_{2}(b-a)(d-c)}{\left(p_{1}+q_{1}\right)\left(p_{2}+q_{2}\right)}\left(\int_{0}^{1} \int_{0}^{1}\left|\left(1-\left(p_{1}+q_{1}\right) z\right)\left(1-\left(p_{2}+q_{2}\right) w\right)\right|^{r_{1}}{ }_{0} d_{p_{1}, q_{1}} z_{0} d_{p_{2}, q_{2}} w\right)^{\frac{1}{r_{1}}} \\
& \times\left(\operatorname { s u p } \left\{\left|\frac{a, c \partial_{p_{1} p_{2}, q_{1} q_{2}}^{2} h(a, c)}{a \partial_{p_{1}, q_{1}} z_{c} \partial_{p_{2}, q_{2}} w}\right|^{r_{2}},\left|\frac{a, c \partial_{p_{1} p_{2}, q_{1} q_{2}}^{2} h(a, d)}{{ }_{a} \partial_{p_{1}, q_{1}} z_{c} \partial_{p_{2}, q_{2}} w}\right|^{r_{2}},\right.\right. \\
& \left.\left.\left|\frac{a_{1}, \partial_{p_{1} p_{2}, q_{1} q_{2}}^{2} h(b, c)}{{ }_{a} \partial_{p_{1}, q_{1}} z_{c} \partial_{p_{2}, q_{2}} w}\right|^{r_{2}},\left|\frac{a, c \partial_{p_{1} p_{2}, q_{1} q_{2}}^{2} h(b, d)}{{ }_{a} \partial_{p_{1}, q_{1}} z_{c} \partial_{p_{2}, q_{2}} w}\right|^{r_{2}}\right\}\right)^{\frac{1}{r_{2}}} .
\end{aligned}
$$

Now, using the properties of the supremum and Definition 3, we get the desired result.

Remark 5. 1. Setting $q_{i} \rightarrow 1^{-}$and $p_{i}=1$ for $i=1,2$ in Theorems 10 and 11 , we get the corresponding results of the classical calculus of the functions of two variables.

2. Furthermore, if $p_{i}=1$ for $i=1,2$ in Theorems 10 and 11 , then Theorems 10 and 11 reduce to Theorems 8 and 9 proven in [22].

\section{Conclusions}

In this paper, we acquired some new results of the Hermit-Hadamard-type inequalities using the $(p, q)$-calculus of the functions of two variables that extend the Hermit-Hadamard-type inequality proven in [22]. In this work, we generalized the results given in [22] and contained the results of the Hermit-Hadamard-type inequalities of the functions of two variables using the usual calculus as special cases when $p=1$ and $q \rightarrow 1^{-}$. The concepts and techniques discussed in this article are excepted to stimulate further research in the field of the $(p, q)$-calculus of the functions of two variables.

Author Contributions: Conceptualization, H.K. and M.A.; writing, original draft preparation, H.K.; writing, review and editing, S.H., M.-u.-D.J., and G.S.; supervision, M.A.

Funding: This research work is funded by the School of Mathematical Sciences, Zhejiang University, Hangzhou 310027, PR-China.

Acknowledgments: The Chinese Government is acknowledged for providing a full scholarship for PhD studies to Miss Humaira Kalsoom.

Conflicts of Interest: The authors declare that they have no competing interests.

\section{References}

1. Jackson, F.H. On a q-definite integrals. Q. J. Pure Appl. Math. 1910, 4, 193-203.

2. Ernst, T. A Comprehensive Treatment of $q$-Calculus; Springer: Basel, Switzerland, 2012.

3. Gauchman, H. Integral inequalities in q-calculus. Comput. Math. Appl. 2004, 47, 281-300. [CrossRef]

4. Kac, V.; Cheung, P. Quantum Calculus; Springer: Berlin, Germany; New York, NY, USA, 2001.

5. Hadamard, J. Étude sur les Propriétés des Fonctions Entières en Particulier d'une Fonction Considérée par Riemann. J. Math. Pures Appl. 1893, 58, 171-215. (In Spanish)

6. Mitrinović, D.S.; Lacković, I.B. Hermite and convexity. Aequationes Math. 1985, 28, 229-232. [CrossRef]

7. Zafar, F.; Kalsoom, H.; Hussain, N. Some inequalities of Hermite-Hadamard-type for n-times differentiable $(\rho, \mathrm{m})$-geometrically convex functions. J. Nonlinear Sci. Appl. 2015, 8, 201-217. [CrossRef]

8. Tariboon, J.; Ntouyas, S.K. Quantum integral inequalities on finite intervals. J. Inequal. Appl. 2014, 121, 13. [CrossRef]

9. Tariboon, J.; Ntouyas, S.K. Quantum calculus on finite intervals and applications to impulsive difference equations. Adv. Differ. Equ. 2013, 282, 19. [CrossRef]

10. Alp, N.; Sarıkaya, M.Z.; Kunt, M.; İscan, İ. q-Hermite-Hadamard inequalities and quantum estimates for midpoint type inequalities via convex and quasi-convex functions. J. King Saud Univ. Sci. 2018, 30, 193-203. [CrossRef]

11. Kalsoom, H.; Wu, J.; Hussain, S.; Latif, M.A. Simpson's type inequalities for coordinated convex functions on quantum calculus. Symmetry 2019, 11, 768. [CrossRef] 
12. Noor, M.A.; Noor, K.I.; Awan, M.U. Some quantum estimates for Hermit-Hadamard inequalities. Appl. Math. Comput. 2015, 251, 675-679.

13. Noor, M.A.; Noor, K.I.; Awan, M.U. Some quantum integral inequalities via preinvex functions. Appl. Math. Comput. 2015, 269, 242-251. [CrossRef]

14. Sudsutad, W.; Ntouyas, S.K.; Tariboon, J. Quantum integral inequalities for convex functions. J. Math. Inequal. 2015, 9, 781-793. [CrossRef]

15. Liu, W.; Zhuang, H. Some quantum estimates of Hermit-Hadamard inequalities for convex functions. J. Appl. Anal. Comput. 2017, 7, 501-522.

16. Zhuang, H.; Liu, W.; Park, J. Some quantum estimates of Hermite-Hadmard inequalities for quasi-convex functions. Mathematics 2019, 7, 152. [CrossRef]

17. Tunç, M.; Göv, E. ( $p, q)$-Integral inequalities. RGMIA Res. Rep. Coll. 2016, 19, 1-13.

18. Tunç, M.; Göv, E. Some integral inequalities via $(p, q)$-calculus on finite intervals. RGMIA Res. Rep. Coll. 2016, 19, 1-12.

19. Tunç, M.; Göv, E. ( $p, q)$-integral inequalities for convex functions. RGMIA Res. Rep. Coll. 2016, 19, 1-12.

20. Dragomir, S.S. On the Hadamard's inequality for functions on the coordinates in a rectangle from the plane. Taiwanese J. Math. 2001, 4, 775-788. [CrossRef]

21. Kunt, M.; İşcan, İ.; Alp, N.; Sarikaya, M.Z. Hermit-Hadamard inequalities and $(p, q)$-estimates for midpoint type inequalities via convex and quasi-convex functions. RACSAM 2018, 112, 969-992. [CrossRef]

22. Latif, M.A.; Dragomir, S.S.; Momoniat, E. Some $q$-analogues of Hermit-Hadamard inequality of functions of two variables on finite rectangles in the plane. J. King Saud Univ. Sci. 2017, 29, 263-273. [CrossRef]

23. Alp, N.; Sarıkaya, M.Z. Hadamard's Inequality for Functions on the Coordinates Convex Functions on Quantum Integral. Available online: https://www.researchgate.net/publication/329814839_ HERMITE_HADAMARD'S_TYPE_INEQUALITIES_FOR_CO-ORDINATED_CONVEX_FUNCTIONS_ ON_QUANTUM_INTEGRAL (accessed on 29 June 2019).

24. Duran, U.; Acikgoz, M.; Araci, S. On generalized some ( $p, q)$-special polynomials. J. Math. Stat. 2018, 14, 129-140. [CrossRef]

25. Sarikaya, M.Z.; Set, E.; Özdemir, M.E.; Dragomir, S.S. New some Hadamard's type inequalities for coordinated convex functions. Tamsui Oxf. J. Inf. Math. Sci. 2012, 28, 137-152.

(C) 2019 by the authors. Licensee MDPI, Basel, Switzerland. This article is an open access article distributed under the terms and conditions of the Creative Commons Attribution (CC BY) license (http:/ / creativecommons.org/licenses/by/4.0/). 